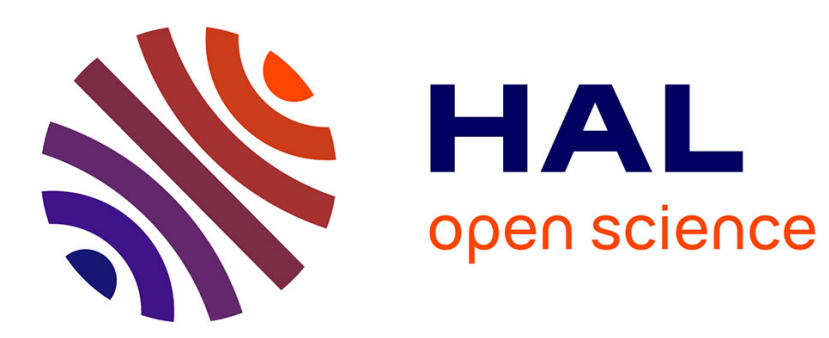

\title{
Roadmap for preferential logics
}

\author{
Dov Gabbay, Karl Schlechta
}

\section{- To cite this version:}

Dov Gabbay, Karl Schlechta. Roadmap for preferential logics. 2008. hal-00311941

\section{HAL Id: hal-00311941 \\ https://hal.science/hal-00311941}

Preprint submitted on 22 Aug 2008

HAL is a multi-disciplinary open access archive for the deposit and dissemination of scientific research documents, whether they are published or not. The documents may come from teaching and research institutions in France or abroad, or from public or private research centers.
L'archive ouverte pluridisciplinaire HAL, est destinée au dépôt et à la diffusion de documents scientifiques de niveau recherche, publiés ou non, émanant des établissements d'enseignement et de recherche français ou étrangers, des laboratoires publics ou privés. 


\title{
Roadmap for preferential logics
}

\author{
Dov M Gabbay* \\ King's College, London ${ }^{\dagger}$ \\ Karl Schlechta $\ddagger$ \\ Laboratoire d'Informatique Fondamentale de Marseille ${ }^{\S}$
}

August 22, 2008

\section{Contents}

1 Introduction 2

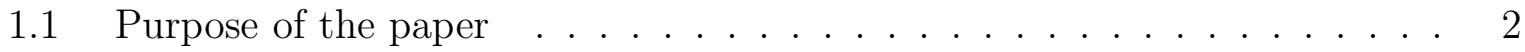

1.2 Organisation of the paper . . . . . . . . . . . . . 2

1.3 Summary of the tables . . . . . . . . . . . . . . . 3

2 Generalities $\quad 4$

3 Logical rules $\quad 6$

$4 \quad$ Preferential structures $\quad 28$

4.1 General and smooth preferential structures . . . . . . . . . . . . . . 28

4.1.1 Definitions and basics . . . . . . . . . . . . . . 28

4.1.2 Representation .................... 31

\footnotetext{
*Dov.Gabbay@kcl.ac.uk, www.dcs.kcl.ac.uk/staff/dg

${ }^{\dagger}$ Department of Computer Science, King's College London, Strand, London WC2R 2LS, UK

${ }_{\ddagger}^{\ddagger} \mathrm{ks} @$ cmi.univ-mrs.fr, karl.schlechta@web.de, http://www.cmi.univ-mrs.fr/ ks

§UMR 6166, CNRS and Université de Provence, Address: CMI, 39, rue Joliot-Curie, F-13453 Marseille Cedex 13, France
} 
4.2 Ranked structures . . . . . . . . . . . . . . . . . 33

4.2.1 Definitions and basics . . . . . . . . . . . . . 33

4.2.2 Representation . . . . . . . . . . . . . . 35

5 Theory revision $\quad 38$

5.1 AGM revision . . . . . . . . . . . . . . . . . . 38

5.2 Distance based revision . . . . . . . . . . . . . . . . . . 41

$5.2 .1 \quad$ Definitions and basics . . . . . . . . . . . . . . . 41

5.2 .2 Representation . . . . . . . . . . . . . . . . 42

$\begin{array}{lll}6 & \text { Size } & 48\end{array}$

References $\quad 55$

\section{Introduction}

\subsection{Purpose of the paper}

The purpose of these pages is to give the reader a systematic overview of logical and algebraic rules used in nonmonotonic and related logics. We try to give orientation in a multitude of sometimes quite similar rules, and in translating the different versions to each other.

The emphasis is on systematisation, and we will not go into deeper completeness proofs.

\subsection{Organisation of the paper}

The article is built around several tables.

They show

(1) connections between semantical and proof theoretical rules, but also their (sometimes subtle) differences,

(2) connections between different semantical rules, but again also their (sometimes subtle) differences.

Further tables summarize 
(1) representation results for preferential structures,

(2) connections between the different concepts of AGM revision,

(3) results for distance based theory revision,

(4) connections between filters, the notion of size, and nonmonotonic logic.

The last table is probably the most innovative part of the paper, and it led to the introduction of perhaps new rules (variants of the $(O R)$ rule).

Yet, as tables go, the emphasis is more on systematisation than on novelty.

The "subtle" part of the comparisons and differences concerns mostly domain closure problems:

- is a domain closed under finite union?

- does the operator preserve definability, i.e. is $f(M(T))=M\left(T^{\prime}\right)$ for some $T^{\prime}$ - where $T, T^{\prime}$ are sets of formulas, and $M(T)$ is the set of classical models of $T$ ?

- is the complement of $M(T)$ again some $M\left(T^{\prime}\right)$ ? etc.

Thus, as a good roadmap should, the article points out easy ways to go from $\mathrm{A}$ to $B$, but also puts up warning signs where there are problems ahead.

\subsection{Summary of the tables}

(1) Tables about rules for nonmonotonic logics

- Definition of the rules, Definition 3.3

- Connections between the different semantical rules, Fact 3.6

- Translations between the logical and the semantical variants, Proposition 3.8

(2) Summary of representation by preferential structures, Table 4.1.1

(3) Tables about rules for theory revision

- AGM revision, definitions of logical and semantical versions, Definition 5.1

- Interdefinability of AGM concepts, Proposition 5.2

- Definition of rules for distance based revision, Conditions 5.1

- Translation between semantical and logical versions, Proposition 5.5 
(4) Tables concerning size and coherence

- Definitions of (weak) filters, ideals, and coherence conditions, Definition 6.1

- Correspondence between coherence conditions and semantical rules for nonmonotonic logics, Fact 6.3

\section{Generalities}

\section{Definition 2.1}

We use $\mathcal{P}$ to denote the power set operator, $\Pi\left\{X_{i}: i \in I\right\}:=\left\{g: g: I \rightarrow \bigcup\left\{X_{i}: i \in I\right\}\right.$, $\left.\forall i \in I . g(i) \in X_{i}\right\}$ is the general cartesian product, $\operatorname{card}(X)$ shall denote the cardinality of $X$, and $V$ the set-theoretic universe we work in - the class of all sets. Given a set of pairs $\mathcal{X}$, and a set $X$, we denote by $\mathcal{X}\lceil X:=\{<x, i>\in \mathcal{X}: x \in X\}$. When the context is clear, we will sometime simply write $X$ for $\mathcal{X}\lceil X$.

$A \subseteq B$ will denote that $A$ is a subset of $B$ or equal to $B$, and $A \subset B$ that $A$ is a proper subset of $B$, likewise for $A \supseteq B$ and $A \supset B$.

Given some fixed set $U$ we work in, and $X \subseteq U$, then $C(X):=U-X$.

If $\mathcal{Y} \subseteq \mathcal{P}(X)$ for some $X$, we say that $\mathcal{Y}$ satisfies

$(\cap)$ iff it is closed under finite intersections,

$(\cap)$ iff it is closed under arbitrary intersections,

$(\cup)$ iff it is closed under finite unions,

(U) iff it is closed under arbitrary unions,

$(\boldsymbol{C})$ iff it is closed under complementation.

We will sometimes write $A=B \| C$ for: $A=B$, or $A=C$, or $A=B \cup C$.

We make ample and tacit use of the Axiom of Choice.

\section{Definition 2.2}

$\prec^{*}$ will denote the transitive closure of the relation $\prec$. If a relation $<, \prec$, or similar is given, $a \perp b$ will express that a and $b$ are $<-($ or $\prec-)$ incomparable - context will tell. Given any relation $<, \leq$ will stand for $<$ or $=$, conversely, given $\leq$, $<$ will stand for $\leq$, but not $=$, similarly for $\prec$ etc.

\section{Definition 2.3}

A child (or successor) of an element $x$ in a tree $t$ will be a direct child in $t$. A child of a child, etc. will be called an indirect child. Trees will be supposed to grow downwards, so the root is the top element. 


\section{Definition 2.4}

A subsequence $\sigma_{i}: i \in I \subseteq \mu$ of a sequence $\sigma_{i}: i \in \mu$ is called cofinal, iff for all $i \in \mu$ there is $i^{\prime} \in I i \leq i^{\prime}$.

Given two sequences $\sigma_{i}$ and $\tau_{i}$ of the same length, then their Hamming distance is the quantity of $i$ where they differ. 


\section{$3 \quad$ Logical rules}

\section{Definition 3.1}

We work here in a classical propositional language $\mathcal{L}$, a theory $T$ will be an arbitrary set of formulas. Formulas will often be named $\phi, \psi$, etc., theories $T$, $S$, etc.

$v(\mathcal{L})$ will be the set of propositional variables of $\mathcal{L}$.

$M_{\mathcal{L}}$ will be the set of (classical) models of $\mathcal{L}, M(T)$ or $M_{T}$ is the set of models of $T$, likewise $M(\phi)$ for a formula $\phi$.

$\boldsymbol{D}_{\mathcal{L}}:=\{M(T): T$ a theory in $\mathcal{L}\}$, the set of definable model sets.

Note that, in classical propositional logic, $\emptyset, M_{\mathcal{L}} \in \boldsymbol{D}_{\mathcal{L}}, \boldsymbol{D}_{\mathcal{L}}$ contains singletons, is closed under arbitrary intersections and finite unions.

An operation $f: \mathcal{Y} \rightarrow \mathcal{P}\left(M_{\mathcal{L}}\right)$ for $\mathcal{Y} \subseteq \mathcal{P}\left(M_{\mathcal{L}}\right)$ is called definability preserving, $(d p)$ or $(\mu d p)$ in short, iff for all $X \in \boldsymbol{D}_{\mathcal{L}} \cap \mathcal{Y} f(X) \in \boldsymbol{D}_{\mathcal{L}}$.

We will also use $(\mu d p)$ for binary functions $f: \mathcal{Y} \times \mathcal{Y} \rightarrow \mathcal{P}\left(M_{\mathcal{L}}\right)$ - as needed for theory revision - with the obvious meaning.

$\vdash$ will be classical derivability, and

$\bar{T}:=\{\phi: T \vdash \phi\}$, the closure of $T$ under $\vdash$.

Con(.) will stand for classical consistency, so $\operatorname{Con}(\phi)$ will mean that $\phi$ is clasical consistent, likewise for $\operatorname{Con}(T)$. $\operatorname{Con}\left(T, T^{\prime}\right)$ will stand for $\operatorname{Con}\left(T \cup T^{\prime}\right)$, etc.

Given a consequence relation $\sim$, we define

$\overline{\bar{T}}:=\{\phi: T \sim \phi\}$.

(There is no fear of confusion with $\bar{T}$, as it just is not useful to close twice under classical logic.)

$T \vee T^{\prime}:=\left\{\phi \vee \phi^{\prime}: \phi \in T, \phi^{\prime} \in T^{\prime}\right\}$.

If $X \subseteq M_{\mathcal{L}}$, then $T h(X):=\{\phi: X \models \phi\}$, likewise for $T h(m), m \in M_{\mathcal{L}}$.

We recollect and note:

\section{Fact 3.1}

Let $\mathcal{L}$ be a fixed propositional language, $\boldsymbol{D}_{\mathcal{L}} \subseteq X, \mu: X \rightarrow \mathcal{P}\left(M_{\mathcal{L}}\right)$, for a $\mathcal{L}$-theory $T$ $\overline{\bar{T}}:=T h\left(\mu\left(M_{T}\right)\right)$, let $T, T^{\prime}$ be arbitrary theories, then:

(1) $\mu\left(M_{T}\right) \subseteq M_{\overline{\bar{T}}}$

(2) $M_{T} \cup M_{T^{\prime}}=M_{T \vee T^{\prime}}$ and $M_{T \cup T^{\prime}}=M_{T} \cap M_{T^{\prime}}$,

(3) $\mu\left(M_{T}\right)=\emptyset \leftrightarrow \perp \in \overline{\bar{T}}$. 
If $\mu$ is definability preserving or $\mu\left(M_{T}\right)$ is finite, then the following also hold:

(4) $\mu\left(M_{T}\right)=M_{\overline{\bar{T}}}$

(5) $T^{\prime} \vdash \overline{\bar{T}} \leftrightarrow M_{T^{\prime}} \subseteq \mu\left(M_{T}\right)$,

(6) $\mu\left(M_{T}\right)=M_{T^{\prime}} \leftrightarrow \overline{T^{\prime}}=\overline{\bar{T}}$.

\section{Fact 3.2}

Let $A, B \subseteq M_{\mathcal{L}}$.

Then $\operatorname{Th}(A \cup B)=T h(A) \cap T h(B)$.

\section{Proof}

$\phi \in T h(A \cup B) \Leftrightarrow A \cup B \models \phi \Leftrightarrow A \models \phi$ and $B \models \phi \Leftrightarrow \phi \in T h(A)$ and $\phi \in T h(B)$.

\section{Fact 3.3}

Let $X \subseteq M_{\mathcal{L}}, \phi, \psi$ formulas.

(1) $X \cap M(\phi) \models \psi$ iff $X \models \phi \rightarrow \psi$.

(2) $X \cap M(\phi) \models \psi$ iff $M(T h(X)) \cap M(\phi) \models \psi$.

(3) $T h(X \cap M(\phi))=\overline{T h(X) \cup\{\phi\}}$

(4) $X \cap M(\phi)=\emptyset \Leftrightarrow M(T h(X)) \cap M(\phi)=\emptyset$

(5) $T h\left(M(T) \cap M\left(T^{\prime}\right)\right)=\overline{T \cup T^{\prime}}$.

\section{Proof}

(1) " $\Rightarrow ": X=(X \cap M(\phi)) \cup(X \cap M(\neg \phi))$. In both parts holds $\neg \phi \vee \psi$, so $X \models \phi \rightarrow \psi$. " $\Leftarrow "$ : Trivial.

(2) $X \cap M(\phi) \models \psi$ (by (1)) iff $X \models \phi \rightarrow \psi$ iff $M(T h(X)) \models \phi \rightarrow \psi$ iff (again by (1)) $M(T h(X)) \cap M(\phi) \models \psi$.

(3) $\psi \in T h(X \cap M(\phi)) \Leftrightarrow X \cap M(\phi) \models \psi \Leftrightarrow_{(2)} M(T h(X) \cup\{\phi\})=M(T h(X)) \cap M(\phi) \models \psi$ $\Leftrightarrow T h(X) \cup\{\phi\} \vdash \psi$.

(4) $X \cap M(\phi)=\emptyset \Leftrightarrow X \models \neg \phi \Leftrightarrow{ }_{(1)} M(T h(X)) \models \neg \phi \Leftrightarrow M(T h(X)) \cap M(\phi)=\emptyset$. 
(5) $M(T) \cap M\left(T^{\prime}\right)=M\left(T \cup T^{\prime}\right)$.

\section{Fact 3.4}

If $X=M(T)$, then $M(T h(X))=X$.

\section{Proof}

$X \subseteq M(T h(X))$ is trivial. $T h(M(T))=\bar{T}$ is trivial by classical soundness and completeness. So $M(T h(M(T))=M(\bar{T})=M(T)=X$.

\section{Example 3.1}

If $v(\mathcal{L})$ is infinite, and $m$ any model for $\mathcal{L}$, then $M:=M_{\mathcal{L}}-\{m\}$ is not definable by any theory $T$. (Proof: Suppose it were, and let $\phi$ hold in $M$, but not in $m$, so in $m \neg \phi$ holds, but as $\phi$ is finite, there is a model $m^{\prime}$ in $M$ which coincides on all propositional variables of $\phi$ with $m$, so in $m^{\prime} \neg \phi$ holds, too, a contradiction.) Thus, in the infinite case, $\mathcal{P}\left(M_{\mathcal{L}}\right) \neq \boldsymbol{D}_{\mathcal{L}}$

(There is also a simple cardinality argument, which shows that almost no model sets are definable, but it is not constructive and thus less instructive than above argument. We give it nonetheless: Let $\kappa:=\operatorname{card}(v(\mathcal{L}))$. Then there are $\kappa$ many formulas, so $2^{\kappa}$ many theories, and thus $2^{\kappa}$ many definable model sets. But there are $2^{\kappa}$ many models, so $\left(2^{\kappa}\right)^{\kappa}$ many model sets.)

\section{Definition 3.2}

Let $\mathcal{Y} \subseteq \mathcal{P}(Z)$ be given and closed under arbitrary intersections.

For $A \subseteq Z$, let $\overbrace{A}:=\bigcap\{X \in \mathcal{Y}: A \subseteq X\}$.

Intuitively, $Z$ is the set of all models for $\mathcal{L}, \mathcal{Y}$ is $\boldsymbol{D}_{\mathcal{L}}$, and $\overbrace{A}=M(T h(A))$, this is the intended application. Note that then $\overbrace{\emptyset}=\emptyset$.

\section{Fact 3.5}


(1) If $\mathcal{Y} \subseteq \mathcal{P}(Z)$ is closed under arbitrary intersections and finite unions, $Z \in \mathcal{Y}, X, Y \subseteq Z$, then the following hold:

$(C l \cup) \overbrace{X \cup Y}=\overbrace{X} \cup \overbrace{Y}$

$(C l \cap) \overbrace{X \cap Y} \subseteq \overbrace{X} \cap \overbrace{Y}$, but usually not conversely,

$(C l-) \overbrace{A}-\overbrace{B}^{\subseteq} \subseteq \overbrace{A-B}$,

$(C l=) X=Y \rightarrow \overbrace{X}=\overbrace{Y}$, but not conversely,

$(C l \subseteq 1) \overbrace{X} \subseteq Y \rightarrow X \subseteq Y$, but not conversely,

$(C l \subseteq 2) X \subseteq \overbrace{Y} \rightarrow \overbrace{X}^{\subseteq} \subseteq \overbrace{Y}$.

(2) If, in addition, $X \in \mathcal{Y}$ and $C X:=Z-X \in \mathcal{Y}$, then the following two properties hold, too:

$(C l \cap+) \overbrace{A}^{A} \cap X=\overbrace{A \cap X}$,

$(C l-+) \overbrace{A}-X=\overbrace{A-X}$.

(3) In the intended application, i.e. $\overbrace{A}=M(T h(A))$, the following hold:

(3.1) $\operatorname{Th}(X)=T h(\overbrace{X})$,

(3.2) Even if $A=\overbrace{A}, B=\overbrace{B}$, it is not necessarily true that $\overbrace{A-B} \subseteq \overbrace{A}-\overbrace{B}$.

\section{Proof:}

$(C l=),(C l \subseteq 1),(C l \subseteq 2),(3.1)$ are trivial.

$(C l \cup)$ Let $\mathcal{Y}(U):=\{X \in \mathcal{Y}: U \subseteq X\}$. If $A \in \mathcal{Y}(X \cup Y)$, then $A \in \mathcal{Y}(X)$ and $A \in \mathcal{Y}(Y)$, so $\overbrace{X \cup Y} \supseteq \overbrace{X} \cup \overbrace{Y}$. If $A \in \mathcal{Y}(X)$ and $B \in \mathcal{Y}(Y)$, then $A \cup B \in \mathcal{Y}(X \cup Y)$, so $\overbrace{X \cup Y}$ $\subseteq \overbrace{X}^{X} \cup \overbrace{Y}^{\varrho}$.

$(C l \cap)$ Let $X^{\prime}, Y^{\prime} \in \mathcal{Y}, X \subseteq X^{\prime}, Y \subseteq Y^{\prime}$, then $X \cap Y \subseteq X^{\prime} \cap Y^{\prime}$, so $\overbrace{X \cap Y} \subseteq \overbrace{X} \cap \overbrace{Y}$. For the converse, set $X:=M_{\mathcal{L}}-\{m\}, Y:=\{m\}$ in Example 3.1.

$(C l-)$ Let $A-B \subseteq X \in \mathcal{Y}, B \subseteq Y \in \mathcal{Y}$, so $A \subseteq X \cup Y \in \mathcal{Y}$. Let $x \notin \overbrace{B} \Rightarrow$ $\exists Y \in \mathcal{Y}(B \subseteq Y, x \notin Y), x \notin \overbrace{A-B}^{B-B} \Rightarrow \exists \in \mathcal{Y}(A-B \subseteq X, x \notin X)$, so $x \notin X \cup Y$, $A \subseteq X \cup Y$, so $x \notin \overbrace{A}$. Thus, $x \notin \overbrace{B}, x \notin \overbrace{A-B} \Rightarrow x \notin \overbrace{A}$, or $x \in \overbrace{A}-\overbrace{B} \Rightarrow$ $x \in \overbrace{A-B}$.

$(C l \cap+) \overbrace{A} \cap X \supseteq \overbrace{A \cap X}$ by $(C l \cap)$. For " $\subseteq$ ": Let $A \cap X \subseteq A^{\prime} \in \mathcal{Y}$, then by closure 
under $(\cup), A \subseteq A^{\prime} \cup \boldsymbol{C} X \in \mathcal{Y},\left(A^{\prime} \cup \boldsymbol{C} X\right) \cap X \subseteq A^{\prime}$. So $\overbrace{A} \cap X \subseteq \overbrace{A \cap X}$.

$(C l-+) \overbrace{A-X}=\overbrace{A \cap \boldsymbol{C} X}=\overbrace{A}^{A} \cap \boldsymbol{C} X=\overbrace{A}^{A}-X$ by $(C l \cap+)$.

(3.2) Set $A:=M_{\mathcal{L}}, B:=\{m\}$ for $m \in M_{\mathcal{L}}$ arbitrary, $\mathcal{L}$ infinite. So $A=\overbrace{A}, B=\overbrace{B}$, but $\overbrace{A-B}=A \neq A-B$.

\section{Definition 3.3}

We introduce here formally a list of properties of set functions on the algebraic side, and their corresponding logical rules on the other side.

Recall that $\bar{T}:=\{\phi: T \vdash \phi\}, \overline{\bar{T}}:=\{\phi: T \sim \phi\}$, where $\vdash$ is classical consequence, and $\sim$ any other consequence.

We show, wherever adequate, in parallel the formula version in the left column, the theory version in the middle column, and the semantical or algebraic counterpart in the right column. The algebraic counterpart gives conditions for a function $f: \mathcal{Y} \rightarrow \mathcal{P}(U)$, where $U$ is some set, and $\mathcal{Y} \subseteq \mathcal{P}(U)$.

When the formula version is not commonly used, we omit it, as we normally work only with the theory version.

Intuitively, $A$ and $B$ in the right hand side column stand for $M(\phi)$ for some formula $\phi$, whereas $X, Y$ stand for $M(T)$ for some theory $T$. 


\begin{tabular}{|c|c|c|}
\hline \multicolumn{3}{|c|}{ Basics } \\
\hline $\begin{array}{c}(A N D) \\
\phi \sim \psi, \phi \sim \psi^{\prime} \Rightarrow \\
\phi \sim \psi \wedge \psi^{\prime}\end{array}$ & $\begin{array}{c}(A N D) \\
T \sim \psi, T \sim \psi^{\prime} \Rightarrow \\
T \sim \psi \wedge \psi^{\prime}\end{array}$ & $\begin{array}{l}\text { Closure under } \\
\text { finite } \\
\text { intersection }\end{array}$ \\
\hline $\begin{array}{c}(O R) \\
\phi \sim \psi, \phi^{\prime} \sim \psi \Rightarrow \\
\phi \vee \phi^{\prime} \sim \psi\end{array}$ & $\begin{array}{c}\overline{\bar{T}} \cap \overline{(O R)} \overline{\overline{T^{\prime}}} \subseteq \overline{\overline{T \vee T^{\prime}}}\end{array}$ & $\begin{array}{c}(\mu O R) \\
f(X \cup Y) \subseteq f(X) \cup f(Y)\end{array}$ \\
\hline $\begin{array}{c}(w O R) \\
\phi \sim \psi, \phi^{\prime} \vdash \psi \Rightarrow \\
\phi \vee \phi^{\prime} \sim \psi\end{array}$ & $\begin{array}{c}\overline{\bar{T}} \cap \overline{T^{\prime}} \subseteq \overline{\overline{T \vee T^{\prime}}} \\
\end{array}$ & $\begin{array}{c}(\mu w O R) \\
f(X \cup Y) \subseteq f(X) \cup Y\end{array}$ \\
\hline $\begin{array}{c}(\operatorname{disjOR)} \\
\phi \vdash \neg \phi^{\prime}, \phi \sim \psi, \\
\phi^{\prime} \sim \psi \Rightarrow \phi \vee \phi^{\prime} \sim \psi \\
\end{array}$ & $\begin{array}{c}(\operatorname{disjOR}) \\
\neg \operatorname{Con}\left(T \cup T^{\prime}\right) \Rightarrow \\
\overline{\bar{T}} \cap \overline{\overline{T^{\prime}}} \subseteq \overline{\overline{T \vee T^{\prime}}} \\
\end{array}$ & $\begin{array}{c}(\mu \operatorname{disjOR)} \\
X \cap Y=\emptyset \Rightarrow \\
f(X \cup Y) \subseteq f(X) \cup f(Y)\end{array}$ \\
\hline $\begin{array}{c}(L L E) \\
\text { Left Logical Equivalence } \\
\vdash \phi \leftrightarrow \phi^{\prime}, \phi \sim \psi \Rightarrow \\
\phi^{\prime} \sim \psi\end{array}$ & $\begin{array}{c}(L L E) \\
\bar{T}=\overline{T^{\prime}} \Rightarrow \overline{\bar{T}}=\overline{\overline{T^{\prime}}}\end{array}$ & trivially true \\
\hline $\begin{array}{c}(R W) \text { Right Weakening } \\
\phi \sim \psi, \vdash \psi \rightarrow \psi^{\prime} \Rightarrow \\
\phi \sim \psi^{\prime}\end{array}$ & $\begin{array}{c}(R W) \\
T \sim \psi, \vdash \psi \rightarrow \psi^{\prime} \Rightarrow \\
T \sim \psi^{\prime}\end{array}$ & upward closure \\
\hline$(C C L)$ Classical Closure & $\begin{array}{c}(C C L) \\
\overline{\bar{T}} \text { is classically } \\
\text { closed }\end{array}$ & trivially true \\
\hline $\begin{array}{c}\text { (SC) Supraclassicality } \\
\phi \vdash \psi \Rightarrow \phi \sim \psi \\
(R E F) \text { Reflexivity } \\
\Delta, \alpha \sim \alpha\end{array}$ & $\begin{array}{c}(S C) \\
\bar{T} \subseteq \overline{\bar{T}}\end{array}$ & $\begin{array}{c}(\mu \subseteq) \\
f(X) \subseteq X\end{array}$ \\
\hline $\begin{array}{c}(C P) \\
\text { Consistency Preservation } \\
\phi \sim \perp \Rightarrow \phi \vdash \perp \\
\end{array}$ & $\begin{array}{c}(C P) \\
T \sim \perp \Rightarrow T \vdash \perp\end{array}$ & $\begin{array}{c}(\mu \emptyset) \\
f(X)=\emptyset \Rightarrow X=\emptyset\end{array}$ \\
\hline & & $\begin{array}{c}(\mu \emptyset \text { fin }) \\
X \neq \emptyset \Rightarrow f(X) \neq \emptyset \\
\text { for finite } X\end{array}$ \\
\hline$\overline{\overline{\phi \wedge \phi^{\prime}}} \subseteq \overline{\overline{\bar{\phi} \cup\left\{\phi^{\prime}\right\}}}$ & $\begin{array}{c}(P R) \\
\overline{\overline{T \cup T^{\prime}}} \subseteq \overline{\bar{T} \cup T^{\prime}}\end{array}$ & $\begin{array}{c}(\mu P R) \\
X \subseteq Y \Rightarrow \\
f(Y) \cap X \subseteq f(X)\end{array}$ \\
\hline & & $\begin{array}{c}\left(\mu P R^{\prime}\right) \\
f(X) \cap Y \subseteq f(X \cap Y)\end{array}$ \\
\hline $\begin{array}{c}(C U T) \\
\Delta \sim \alpha ; \Delta, \alpha \sim \beta \Rightarrow \\
\Delta \mid \sim \beta\end{array}$ & 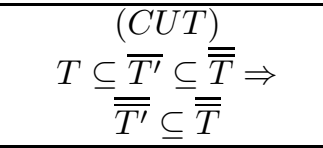 & $\begin{array}{c}(\mu C U T) \\
f(X) \subseteq Y \subseteq X \Rightarrow \\
f(X) \subseteq f(Y)\end{array}$ \\
\hline
\end{tabular}




\begin{tabular}{|c|c|c|}
\hline \multicolumn{3}{|c|}{ Cumulativity } \\
\hline$(C M)$ Cautious Monotony & \multirow{4}{*}{$\begin{array}{c}(C M) \\
T \subseteq \overline{T^{\prime}} \subseteq \overline{\bar{T}} \\
\overline{\bar{T}} \subseteq \overline{\overline{T^{\prime}}}\end{array} \Rightarrow$} & $(\mu C M)$ \\
\hline$\phi|\sim \psi, \phi| \sim \psi^{\prime} \Rightarrow$ & & $f(X) \subseteq Y \subseteq X \Rightarrow$ \\
\hline$\phi \wedge \psi \mid \sim \psi^{\prime}$ & & $f(Y) \subseteq f(X)$ \\
\hline $\begin{array}{c}\text { or (Res } M) \text { Restricted Monotony } \\
\Delta \sim \alpha, \beta \Rightarrow \Delta, \alpha \sim \beta\end{array}$ & & $\begin{array}{c}(\mu \operatorname{Res} M) \\
f(X) \subseteq A \cap B \Rightarrow f(X \cap A) \subseteq B\end{array}$ \\
\hline$(C U M)$ Cumulativity & \multirow{2}{*}{$\begin{array}{c}(C U M) \\
T \subseteq \overline{T^{\prime}} \subseteq \overline{\bar{T}} \Rightarrow \\
\overline{\bar{T}}=\overline{\overline{T^{\prime}}}\end{array}$} & $(\mu C U M)$ \\
\hline $\begin{array}{c}\phi \sim \psi \Rightarrow \\
\left(\phi \sim \psi^{\prime} \Leftrightarrow \phi \wedge \psi \sim \psi^{\prime}\right)\end{array}$ & & $\begin{array}{c}f(X) \subseteq Y \subseteq X \Rightarrow \\
f(Y)=f(X)\end{array}$ \\
\hline & $\begin{array}{c}(\subseteq \supseteq) \\
T \subseteq \overline{\overline{T^{\prime}}}, T^{\prime} \subseteq \overline{\bar{T}} \Rightarrow \\
\overline{\overline{T^{\prime}}}=\overline{\bar{T}}\end{array}$ & $\begin{array}{c}(\mu \subseteq \supseteq) \\
f(X) \subseteq Y, f(Y) \subseteq X \Rightarrow \\
f(X)=f(Y)\end{array}$ \\
\hline \multicolumn{3}{|c|}{ Rationality } \\
\hline \multirow[t]{15}{*}{$\begin{array}{c}\text { Rat } M \text { ) Rational Monotony } \\
\phi \sim \psi, \phi \mid \psi \neg \psi^{\prime} \Rightarrow \\
\phi \wedge \psi^{\prime} \sim \psi \\
\end{array}$} & 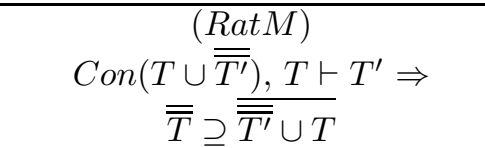 & $\begin{array}{c}(\mu \operatorname{Rat} M) \\
X \subseteq Y, X \cap f(Y) \neq \emptyset \Rightarrow \\
f(X) \subseteq f(Y) \cap X\end{array}$ \\
\hline & $(\operatorname{Rat} M=)$ & $(\mu=)$ \\
\hline & $\begin{array}{c}\operatorname{Con}\left(T \cup \overline{\overline{T^{\prime}}}\right), T \vdash T^{\prime} \Rightarrow \\
\overline{\bar{T}}=\overline{\overline{\overline{T^{\prime}}} \cup T}\end{array}$ & $\begin{array}{c}X \subseteq Y, X \cap f(Y) \neq \emptyset \Rightarrow \\
f(X)=f(Y) \cap X\end{array}$ \\
\hline & $\left(\log =^{\prime}\right)$ & $\left(\mu==^{\prime}\right)$ \\
\hline & $\operatorname{Con}\left(\overline{\overline{T^{\prime}}} \cup T\right) \Rightarrow$ & $f(Y) \cap X \neq \emptyset \Rightarrow$ \\
\hline & $\overline{\overline{T \cup T^{\prime}}}=\overline{\overline{\overline{T^{\prime}}} \cup T}$ & $f(Y \cap X)=f(Y) \cap X$ \\
\hline & $(\log \|)$ & $(\mu \|)$ \\
\hline & $\overline{\overline{T \vee T^{\prime}}}$ is one of & $f(X \cup Y)$ is one of \\
\hline & $\overline{\bar{T}}$, or $\overline{\overline{T^{\prime}}}$, or $\overline{\bar{T}} \cap \overline{\overline{T^{\prime}}}($ by $(\mathrm{CCL}))$ & $f(X), f(Y)$ or $f(X) \cup f(Y)$ \\
\hline & $(\log \cup)$ & $(\mu \cup)$ \\
\hline & $\operatorname{Con}\left(\overline{\overline{T^{\prime}}} \cup T\right), \neg \operatorname{Con}\left(\overline{\overline{T^{\prime}}} \cup \overline{\bar{T}}\right) \Rightarrow$ & $f(Y) \cap(X-f(X)) \neq \emptyset \Rightarrow$ \\
\hline & $\neg \operatorname{Con}\left(\overline{\overline{T \vee T^{\prime}}} \cup T^{\prime}\right)$ & $f(X \cup Y) \cap Y=\emptyset$ \\
\hline & $\left(\log \cup^{\prime}\right)$ & $\left(\mu \cup^{\prime}\right)$ \\
\hline & $\begin{array}{c}\operatorname{Con}\left(\overline{\overline{T^{\prime}}} \cup T\right), \neg \operatorname{Con}\left(\overline{\overline{T^{\prime}}} \cup \overline{\bar{T}}\right) \Rightarrow \\
\overline{\overline{T \vee T^{\prime}}}=\overline{\bar{T}}\end{array}$ & $\begin{array}{c}f(Y) \cap(X-f(X)) \neq \emptyset \Rightarrow \\
f(X \cup Y)=f(X)\end{array}$ \\
\hline & & $\begin{array}{c}(\mu \in) \\
a \in X-f(X) \Rightarrow \\
\exists b \in X . a \notin f(\{a, b\})\end{array}$ \\
\hline
\end{tabular}

$(P R)$ is also called infinite conditionalization - we choose the name for its central role for preferential structures $(P R)$ or $(\mu P R)$.

The system of rules $(A N D)(O R)(L L E)(R W)(S C)(C P)(C M)(C U M)$ is also called system $P$ (for preferential), adding (Rat $M)$ gives the system $R$ (for rationality or rankedness).

Roughly: Smooth preferential structures generate logics satisfying system $P$, ranked structures logics satisfying system $R$.

A logic satisfying $(R E F),(\operatorname{Res} M)$, and $(C U T)$ is called a consequence relation. 
$(L L E)$ and $(C C L)$ will hold automatically, whenever we work with model sets.

$(A N D)$ is obviously closely related to filters, and corresponds to closure under finite intersections. $(R W)$ corresponds to upward closure of filters.

More precisely, validity of both depend on the definition, and the direction we consider.

Given $f$ and $(\mu \subseteq), f(X) \subseteq X$ generates a pricipal filter: $\left\{X^{\prime} \subseteq X: f(X) \subseteq X^{\prime}\right\}$, with the definition: If $X=M(T)$, then $T \sim \phi$ iff $f(X) \subseteq M(\phi)$. Validity of $(A N D)$ and $(R W)$ are then trivial.

Conversely, we can define for $X=M(T)$

$\mathcal{X}:=\left\{X^{\prime} \subseteq X: \exists \phi\left(X^{\prime}=X \cap M(\phi)\right.\right.$ and $\left.\left.T \sim \phi\right)\right\}$.

$(A N D)$ then makes $\mathcal{X}$ closed under finite intersections, $(R W)$ makes $\mathcal{X}$ upward closed. This is in the infinite case usually not yet a filter, as not all subsets of $X$ need to be definable this way. In this case, we complete $\mathcal{X}$ by adding all $X^{\prime \prime}$ such that there is $X^{\prime} \subseteq X^{\prime \prime} \subseteq X, X^{\prime} \in \mathcal{X}$.

Alternatively, we can define

$\mathcal{X}:=\left\{X^{\prime} \subseteq X: \bigcap\{X \cap M(\phi): T \sim \phi\} \subseteq X^{\prime}\right\}$.

$(S C)$ corresponds to the choice of a subset.

$(C P)$ is somewhat delicate, as it presupposes that the chosen model set is non-empty. This might fail in the presence of ever better choices, without ideal ones; the problem is addressed by the limit versions.

$(P R)$ is an infinitary version of one half of the deduction theorem: Let $T$ stand for $\phi, T^{\prime}$ for $\psi$, and $\phi \wedge \psi \sim \sigma$, so $\phi \sim \psi \rightarrow \sigma$, but $(\psi \rightarrow \sigma) \wedge \psi \vdash \sigma$.

$(C U M)$ (whose most interesting half in our context is $(C M)$ ) may best be seen as normal use of lemmas: We have worked hard and found some lemmas. Now we can take a rest, and come back again with our new lemmas. Adding them to the axioms will neither add new theorems, nor prevent old ones to hold.

\section{Fact 3.6}

This table is to be read as follows: If the left hand side holds for some function $f: \mathcal{Y} \rightarrow$ $\mathcal{P}(U)$, and the auxiliary properties noted in the middle also hold for $f$ or $\mathcal{Y}$, then the right hand side will hold, too - and conversely. 


\begin{tabular}{|c|c|c|c|}
\hline \multicolumn{4}{|c|}{ Basics } \\
\hline (1.1) & \multirow[t]{2}{*}{$(\mu P R)$} & $\Rightarrow(\cap)+(\mu \subseteq)$ & \multirow[t]{2}{*}{$\left(\mu P R^{\prime}\right)$} \\
\hline$(1.2)$ & & $\Leftarrow$ & \\
\hline$(2.1)$ & \multirow[t]{2}{*}{$(\mu P R)$} & $\Rightarrow(\mu \subseteq)$ & \multirow[t]{2}{*}{$(\mu O R)$} \\
\hline$(2.2)$ & & $\begin{array}{l}\Leftarrow(\mu \subseteq)+\text { closure } \\
\text { under set difference }\end{array}$ & \\
\hline (3) & $(\mu P R)$ & $\Rightarrow$ & $(\mu C U T)$ \\
\hline$(4)$ & $\begin{array}{c}(\mu \subseteq)+(\mu \subseteq \supseteq)+(\mu C U M)+ \\
(\mu R a t M)+(\cap)\end{array}$ & $\not \Rightarrow$ & $(\mu P R)$ \\
\hline \multicolumn{4}{|c|}{ Cumulativity } \\
\hline$(5.1)$ & \multirow[t]{2}{*}{$(\mu C M)$} & $\Rightarrow(\cap)+(\mu \subseteq)$ & \multirow[t]{2}{*}{$(\mu \operatorname{Res} M)$} \\
\hline$(5.2)$ & & $\Leftarrow$ (infin.) & \\
\hline$(6)$ & $(\mu C M)+(\mu C U T)$ & $\Leftrightarrow$ & $(\mu C U M)$ \\
\hline$(7)$ & $(\mu \subseteq)+(\mu \subseteq \supseteq)$ & $\Rightarrow$ & $(\mu C U M)$ \\
\hline$(8)$ & $(\mu \subseteq)+(\mu C U M)+(\cap)$ & $\Rightarrow$ & $(\mu \subseteq \supseteq)$ \\
\hline$(9)$ & $(\mu \subseteq)+(\mu C U M)$ & $\not \Rightarrow$ & $(\mu \subseteq \supseteq)$ \\
\hline \multicolumn{4}{|c|}{ Rationality } \\
\hline$(10)$ & $(\mu R a t M)+(\mu P R)$ & $\Rightarrow$ & $(\mu=)$ \\
\hline$(11)$ & $(\mu=)$ & $\Rightarrow$ & $(\mu P R)$ \\
\hline$(12.1)$ & \multirow[t]{2}{*}{$(\mu=)$} & $\Rightarrow(\cap)+(\mu \subseteq)$ & \multirow[t]{2}{*}{$\left(\mu=^{\prime}\right)$} \\
\hline$(12.2)$ & & $\Leftarrow$ & \\
\hline$(13)$ & $(\mu \subseteq),(\mu=)$ & $\Rightarrow(\cup)$ & $(\mu \cup)$ \\
\hline$(14)$ & $(\mu \subseteq),(\mu \emptyset),(\mu=)$ & $\Rightarrow(\cup)$ & $(\mu \|),\left(\mu \cup^{\prime}\right),(\mu C U M)$, \\
\hline$(15)$ & $(\mu \subseteq)+(\mu \|)$ & $\Rightarrow \mathcal{Y}$ closed under set difference & $(\mu=)$, \\
\hline$(16)$ & $\begin{array}{c}(\mu \|)+(\mu \in)+(\mu P R)+ \\
(\mu \subseteq)\end{array}$ & $\Rightarrow(\cup)+\mathcal{Y}$ contains singletons & $(\mu=)$, \\
\hline$(17)$ & $(\mu C U M)+(\mu=)$ & $\Rightarrow(\cup)+\mathcal{Y}$ contains singletons & $(\mu \in)$, \\
\hline$(18)$ & $(\mu C U M)+(\mu=)+(\mu \subseteq)$ & $\Rightarrow(\cup)$ & $(\mu \|)$, \\
\hline$(19)$ & $(\mu P R)+(\mu C U M)+(\mu \|)$ & $\Rightarrow$ sufficient, e.g. true in $\boldsymbol{D}_{\mathcal{L}}$ & $(\mu=)$. \\
\hline$(20)$ & $(\mu \subseteq)+(\mu P R)+(\mu=)$ & $\not \Rightarrow$ & $(\mu \|)$, \\
\hline$(21)$ & $(\mu \subseteq)+(\mu P R)+(\mu \|)$ & $\begin{array}{l}\not \Rightarrow \text { (without closure } \\
\text { under set difference), }\end{array}$ & $(\mu=)$ \\
\hline$(22)$ & $\begin{array}{c}(\mu \subseteq)+(\mu P R)+(\mu \|)+ \\
(\mu=)+(\mu \cup)\end{array}$ & $\not \Rightarrow$ & $\begin{array}{c}(\mu \in) \\
\text { (thus not representability } \\
\text { by ranked structures) }\end{array}$ \\
\hline
\end{tabular}

\section{Proof}

All sets are to be in $\mathcal{Y}$.

(1.1) $(\mu P R)+(\cap)+(\mu \subseteq) \Rightarrow\left(\mu P R^{\prime}\right):$

By $X \cap Y \subseteq X$ and $(\mu P R), f(X) \cap X \cap Y \subseteq f(X \cap Y)$. By $(\mu \subseteq) f(X) \cap Y=f(X) \cap X \cap Y$.

(1.2) $\left(\mu P R^{\prime}\right) \Rightarrow(\mu P R)$ : 
Let $X \subseteq Y$, so $X=X \cap Y$, so by $\left(\mu P R^{\prime}\right) f(Y) \cap X \subseteq f(X \cap Y)=f(X)$.

$(2.1)(\mu P R)+(\mu \subseteq) \Rightarrow(\mu O R)$ :

$f(X \cup Y) \subseteq X \cup Y$ by $(\mu \subseteq)$, so $f(X \cup Y)=(f(X \cup Y) \cap X) \cup(f(X \cup Y) \cap Y) \subseteq$ $f(X) \cup f(Y)$.

$(2.2)(\mu O R)+(\mu \subseteq)+$ closure under set difference $\Rightarrow(\mu P R)$ :

Let $X \subseteq Y, X^{\prime}:=Y-X . f(Y) \subseteq f(X) \cup f\left(X^{\prime}\right)$ by $(\mu O R)$, so $f(Y) \cap X \subseteq(f(X) \cap X) \cup$ $\left(f\left(X^{\prime}\right) \cap X\right)=_{(\mu \subseteq)} f(X) \cup \emptyset=f(X)$.

(3) $(\mu P R) \Rightarrow(\mu C U T)$ :

$f(X) \subseteq Y \subseteq X \Rightarrow f(X) \subseteq f(X) \cap Y \subseteq f(Y)$ by $(\mu P R)$.

(4) $(\mu \subseteq)+(\mu \subseteq \supseteq)+(\mu C U M)+(\mu \operatorname{Rat} M)+(\cap) \nRightarrow(\mu P R)$ :

This is shown in Example 3.3.

(5.1) $(\mu C M)+(\cap)+(\mu \subseteq) \Rightarrow(\mu \operatorname{Res} M)$ :

Let $f(X) \subseteq A \cap B$, so $f(X) \subseteq A$, so by $(\mu \subseteq) f(X) \subseteq A \cap X \subseteq X$, so by $(\mu C M)$ $f(A \cap X) \subseteq f(X) \subseteq B$.

(5.2) $(\mu \operatorname{Res} M) \Rightarrow(\mu C M)$ :

We consider here the infinitary version, where all sets can be model sets of infinite theories.

Let $f(X) \subseteq Y \subseteq X$, so $f(X) \subseteq Y \cap f(X)$, so by $(\mu \operatorname{Res} M) f(Y)=f(X \cap Y) \subseteq f(X)$.

(6) $(\mu C M)+(\mu C U T) \Leftrightarrow(\mu C U M)$ :

Trivial.

(7) $(\mu \subseteq)+(\mu \subseteq \supseteq) \Rightarrow(\mu C U M)$ :

Suppose $f(D) \subseteq E \subseteq D$. So by $(\mu \subseteq) f(E) \subseteq E \subseteq D$, so by $(\mu \subseteq \supseteq) f(D)=f(E)$.

(8) $(\mu \subseteq)+(\mu C U M)+(\cap) \Rightarrow(\mu \subseteq \supseteq)$ :

Let $f(D) \subseteq E, f(E) \subseteq D$, so by $(\mu \subseteq) f(D) \subseteq D \cap E \subseteq D, f(E) \subseteq D \cap E \subseteq E$. As $f(D \cap E)$ is defined, so $f(D)=f(D \cap E)=f(E)$ by $(\mu C U M)$.

(9) $(\mu \subseteq)+(\mu C U M) \nRightarrow(\mu \subseteq \supseteq)$ :

This is shown in Example 3.2 .

(10) $(\mu R a t M)+(\mu P R) \Rightarrow(\mu=)$ :

Trivial.

(11) $(\mu=)$ entails $(\mu P R)$ :

Trivial.

$(12.1)(\mu=) \rightarrow\left(\mu=^{\prime}\right):$

Let $f(Y) \cap X \neq \emptyset$, we have to show $f(X \cap Y)=f(Y) \cap X$. By $(\mu \subseteq) f(Y) \subseteq Y$, so 
$f(Y) \cap X=f(Y) \cap(X \cap Y)$, so by $(\mu=) f(Y) \cap X=f(Y) \cap(X \cap Y)=f(X \cap Y)$.

(12.2) $\left(\mu=^{\prime}\right) \rightarrow(\mu=):$

Let $X \subseteq Y, f(Y) \cap X \neq \emptyset$, then $f(X)=f(Y \cap X)=f(Y) \cap X$.

(13) $(\mu \subseteq),(\mu=) \rightarrow(\mu \cup):$

If not, $f(X \cup Y) \cap Y \neq \emptyset$, but $f(Y) \cap(X-f(X)) \neq \emptyset$. By (11), ( $\mu P R)$ holds, so $f(X \cup Y) \cap X \subseteq f(X)$, so $\emptyset \neq f(Y) \cap(X-f(X)) \subseteq f(Y) \cap(X-f(X \cup Y))$, so $f(Y)-f(X \cup Y) \neq \emptyset$, so by $(\mu \subseteq) f(Y) \subseteq Y$ and $f(Y) \neq f(X \cup Y) \cap Y$. But by $(\mu=)$ $f(Y)=f(X \cup Y) \cap Y$, a contradiction.

(14)

$(\mu \subseteq),(\mu \emptyset),(\mu=) \Rightarrow(\mu \|):$

If $X$ or $Y$ or both are empty, then this is trivial. Assume then $X \cup Y \neq \emptyset$, so by $(\mu \emptyset)$ $f(X \cup Y) \neq \emptyset$. By $(\mu \subseteq) f(X \cup Y) \subseteq X \cup Y$, so $f(X \cup Y) \cap X=\emptyset$ and $f(X \cup Y) \cap Y=\emptyset$ together are impossible. Case $1, f(X \cup Y) \cap X \neq \emptyset$ and $f(X \cup Y) \cap Y \neq \emptyset:$ By $(\mu=)$ $f(X \cup Y) \cap X=f(X)$ and $f(X \cup Y) \cap Y=f(Y)$, so by $(\mu \subseteq) f(X \cup Y)=f(X) \cup f(Y)$. Case $2, f(X \cup Y) \cap X \neq \emptyset$ and $f(X \cup Y) \cap Y=\emptyset:$ So by $(\mu=) f(X \cup Y)=f(X \cup Y) \cap X=f(X)$. Case 3, $f(X \cup Y) \cap X=\emptyset$ and $f(X \cup Y) \cap Y \neq \emptyset:$ Symmetrical.

$(\mu \subseteq),(\mu \emptyset),(\mu=) \Rightarrow\left(\mu \cup^{\prime}\right):$

If $X \cup Y=\emptyset$, then $f(X \cup Y)=f(X)=\emptyset$ by $(\mu \subseteq)$. So suppose $X \cup Y \neq \emptyset$. By (13), $f(X \cup Y) \cap Y=\emptyset$, so $f(X \cup Y) \subseteq X$ by $(\mu \subseteq)$. By $(\mu \emptyset), f(X \cup Y) \neq \emptyset$, so $f(X \cup Y) \cap X \neq \emptyset$, and $f(X \cup Y)=f(X)$ by $(\mu=)$.

$(\mu \subseteq),(\mu \emptyset),(\mu=) \Rightarrow(\mu C U M):$

If $Y=\emptyset$, this is trivial by $(\mu \subseteq)$. If $Y \neq \emptyset$, then by $(\mu \emptyset)$ - which is crucial here $-f(Y) \neq \emptyset$, so by $f(Y) \subseteq X f(Y) \cap X \neq \emptyset$, so by $(\mu=) f(Y)=f(Y) \cap X=f(X)$.

$(15)(\mu \subseteq)+(\mu \|) \rightarrow(\mu=):$

Let $X \subseteq Y$, and consider $Y=X \cup(Y-\mathrm{X})$. Then $f(Y)=f(X) \| f(Y-\mathrm{X})$. As $f(Y-$ $X) \cap X=\emptyset, f(Y) \cap X \subseteq f(X)$. If $f(Y) \cap X \neq \emptyset$, then by the same argument $f(X)$ is involved, so $f(X) \subseteq f(Y)$.

$(16)(\mu \|)+(\mu \in)+(\mu P R)+(\mu \subseteq) \rightarrow(\mu=):$

Suppose $X \subseteq Y, x \in f(Y) \cap X$, we have to show $f(Y) \cap X=f(X)$. " $\subseteq$ " is trivial by $(\mu P R)$. " $\supseteq$ ": Assume $a \notin f(Y)$ (by $(\mu \subseteq)$ ), but $a \in f(X)$. By $(\mu \in) \exists b \in Y$. $a \notin f(\{a, b\})$. As $a \in f(X)$, by $(\mu P R), a \in f(\{a, x\})$. By $(\mu \|), f(\{a, b, x\})=f(\{a, x\}) \| f(\{b\})$. As $a \notin f(\{a, b, x\}), f(\{a, b, x\})=f(\{b\})$, so $x \notin f(\{a, b, x\})$, contradicting $(\mu P R)$, as $a, b, x \in Y$.

$(17)(\mu C U M)+(\mu=) \rightarrow(\mu \in):$

Let $a \in X-f(X)$. If $f(X)=\emptyset$, then $f(\{a\})=\emptyset$ by $(\mu C U M)$. If not: Let $b \in f(X)$, then 
$a \notin f(\{a, b\})$ by $(\mu=)$.

(18) $(\mu C U M)+(\mu=)+(\mu \subseteq) \rightarrow(\mu \|):$

By $(\mu C U M), f(X \cup Y) \subseteq X \subseteq X \cup Y \rightarrow f(X)=f(X \cup Y)$, and $f(X \cup Y) \subseteq Y \subseteq X \cup Y$ $\rightarrow f(Y)=f(X \cup Y)$. Thus, if $(\mu \|)$ were to fail, $f(X \cup Y) \nsubseteq X, f(X \cup Y) \nsubseteq Y$, but then by $(\mu \subseteq) f(X \cup Y) \cap X \neq \emptyset$, so $f(X)=f(X \cup Y) \cap X$, and $f(X \cup Y) \cap Y \neq \emptyset$, so $f(Y)=f(X \cup Y) \cap Y$ by $(\mu=)$. Thus, $f(X \cup Y)=(f(X \cup Y) \cap X) \cup(f(X \cup Y) \cap Y)=$ $f(X) \cup f(Y)$.

(19) $(\mu P R)+(\mu C U M)+(\mu \|) \rightarrow(\mu=):$

Suppose $(\mu=)$ does not hold. So, by $(\mu P R)$, there are $X, Y, y$ s.t. $X \subseteq Y, X \cap f(Y) \neq$ $\emptyset, y \in Y-f(Y), y \in f(X)$. Let $a \in X \cap f(Y)$. If $f(Y)=\{a\}$, then by $(\mu C U M)$ $f(Y)=f(X)$, so there must be $b \in f(Y), b \neq a$. Take now $Y^{\prime}$, $Y^{\prime \prime}$ s.t. $Y=Y^{\prime} \cup Y^{\prime \prime}$, $a \in Y^{\prime}, a \notin Y^{\prime \prime}, b \in Y^{\prime \prime}, b \notin Y^{\prime}, y \in Y^{\prime} \cap Y^{\prime \prime}$. Assume now $(\mu \|)$ to hold, we show a contradiction. If $y \notin f\left(Y^{\prime \prime}\right)$, then by $(\mu P R) y \notin f\left(Y^{\prime \prime} \cup\{a\}\right)$. But $f\left(Y^{\prime \prime} \cup\{a\}\right)=$ $f\left(Y^{\prime \prime}\right) \| f(\{a, y\})$, so $f\left(Y^{\prime \prime} \cup\{a\}\right)=f\left(Y^{\prime \prime}\right)$, contradicting $a \in f(Y)$. If $y \in f\left(Y^{\prime \prime}\right)$, then by $f(Y)=f\left(Y^{\prime}\right) \| f\left(Y^{\prime \prime}\right), f(Y)=f\left(Y^{\prime}\right)$, contradiction as $b \notin f\left(Y^{\prime}\right)$.

$(20)(\mu \subseteq)+(\mu P R)+(\mu=) \nRightarrow(\mu \|)$ :

See Example 3.4.

$(21)(\mu \subseteq)+(\mu P R)+(\mu \|) \not \Rightarrow(\mu=):$

See Example 3.5.

$(22)(\mu \subseteq)+(\mu P R)+(\mu \|)+(\mu=)+(\mu \cup) \not \Rightarrow(\mu \in):$

See Example 3.6.

Thus, by Fact 4.11, the conditions do not assure representability by ranked structures.

\section{Example 3.2}

We show here $(\mu \subseteq)+(\mu C U M) \nRightarrow(\mu \subseteq \supseteq)$.

Consider $X:=\{a, b, c\}, Y:=\{a, b, d\}, f(X):=\{a\}, f(Y):=\{a, b\}, \mathcal{Y}:=\{X, Y\}$. (If $f(\{a, b\})$ were defined, we would have $f(X)=f(\{a, b\})=f(Y)$, contradiction. $)$

Obviously, $(\mu \subseteq)$ and $(\mu C U M)$ hold, but not $(\mu \subseteq \supseteq)$.

\section{Example 3.3}


We show here $(\mu \subseteq)+(\mu \subseteq \supseteq)+(\mu C U M)+(\mu R a t M)+(\cap) \not \Rightarrow(\mu P R)$.

Let $U:=\{a, b, c\}$. Let $\mathcal{Y}=\mathcal{P}(U)$. So $(\cap)$ is trivially satisfied. Set $f(X):=X$ for all $X \subseteq U$ except for $f(\{a, b\})=\{b\}$. Obviously, this cannot be represented by a preferential structure and $(\mu P R)$ is false for $U$ and $\{a, b\}$. But it satisfies $(\mu \subseteq),(\mu C U M),(\mu R a t M)$. $(\mu \subseteq)$ is trivial. $(\mu C U M)$ : Let $f(X) \subseteq Y \subseteq X$. If $f(X)=X$, we are done. Consider $f(\{a, b\})=\{b\}$. If $\{b\} \subseteq Y \subseteq\{a, b\}$, then $f(Y)=\{b\}$, so we are done again. It is shown in Fact 3.6, (8) that $(\mu \subseteq \supseteq)$ follows. ( $\mu$ Rat $M)$ : Suppose $X \subseteq Y, X \cap f(Y) \neq \emptyset$, we have to show $f(X) \subseteq f(Y) \cap X$. If $f(Y)=Y$, the result holds by $X \subseteq Y$, so it does if $X=Y$. The only remaining case is $Y=\{a, b\}, X=\{b\}$, and the result holds again.

\section{Example 3.4}

The example shows that $(\mu \subseteq)+(\mu P R)+(\mu=) \not \nRightarrow(\mu \|)$.

Consider the following structure without transitivity: $U:=\{a, b, c, d\}, c$ and $d$ have $\omega$ many copies in descending order $c_{1} \succeq c_{2} \ldots$, etc. $a, b$ have one single copy each. $a \succeq b$, $a \succeq d_{1}, b \succeq a, b \succeq c_{1} .(\mu \|)$ does not hold: $f(U)=\emptyset$, but $f(\{a, c\})=\{a\}, f(\{b, d\})=\{b\}$. $(\mu P R)$ holds as in all preferential structures. $(\mu=)$ holds: If it were to fail, then for some $A \subseteq B, f(B) \cap A \neq \emptyset$, so $f(B) \neq \emptyset$. But the only possible cases for $B$ are now: $(a \in B$, $b, d \notin B)$ or $(b \in B, a, c \notin B)$. Thus, $B$ can be $\{a\},\{a, c\},\{b\},\{b, d\}$ with $f(B)=$ $\{a\},\{a\},\{b\},\{b\}$. If $A=B$, then the result will hold trivially. Moreover, $A$ has to be $\neq \emptyset$. So the remaining cases of $B$ where it might fail are $B=\{a, c\}$ and $\{b, d\}$, and by $f(B) \cap A \neq \emptyset$, the only cases of $A$ where it might fail, are $A=\{a\}$ or $\{b\}$ respectively. So the only cases remaining are: $B=\{a, c\}, A=\{a\}$ and $B=\{b, d\}, A=\{b\}$. In the first case, $f(A)=f(B)=\{a\}$, in the second $f(A)=f(B)=\{b\}$, but $(\mu=)$ holds in both.

\section{Example 3.5}

The example shows that $(\mu \subseteq)+(\mu P R)+(\mu \|) \not \Rightarrow(\mu=)$.

Work in the set of theory definable model sets of an infinite propositional language. Note that this is not closed under set difference, and closure properties will play a crucial role in the argumentation. Let $U:=\left\{y, a, x_{i<\omega}\right\}$, where $x_{i} \rightarrow a$ in the standard topology. For the order, arrange s.t. $y$ is minimized by any set iff this set contains a cofinal subsequence of the $x_{i}$, this can be done by the standard construction. Moreover, let the $x_{i}$ all kill themselves, i.e. with $\omega$ many copies $x_{i}^{1} \succeq x_{i}^{2} \succeq \ldots$. There are no other elements in the 
relation. Note that if $a \notin \mu(X)$, then $a \notin X$, and $X$ cannot contain a cofinal subsequence of the $x_{i}$, as $X$ is closed in the standard topology. (A short argument: suppose $X$ contains such a subsequence, but $a \notin X$. Then the theory of a $T h(a)$ is inconsistent with $T h(X)$, so already a finite subset of $T h(a)$ is inconsistent with $T h(X)$, but such a finite subset will finally hold in a cofinal sequence converging to a.) Likewise, if $y \in \mu(X)$, then $X$ cannot contain a cofinal subsequence of the $x_{i}$.

Obviously, $(\mu \subseteq)$ and $(\mu P R)$ hold, but $(\mu=)$ does not hold: Set $B:=U, A:=\{a, y\}$. Then $\mu(B)=\{a\}, \mu(A)=\{a, y\}$, contradicting $(\mu=)$.

It remains to show that $(\mu \|)$ holds.

$\mu(X)$ can only be $\emptyset,\{a\},\{y\},\{a, y\}$. As $\mu(A \cup B) \subseteq \mu(A) \cup \mu(B)$ by $(\mu P R)$,

Case $1, \mu(A \cup B)=\{a, y\}$ is settled.

Note that if $y \in X-\mu(X)$, then $X$ will contain a cofinal subsequence, and thus $a \in \mu(X)$.

Case 2: $\mu(A \cup B)=\{a\}$.

Case 2.1: $\mu(A)=\{a\}$ - we are done.

Case 2.2: $\mu(A)=\{y\}: A$ does not contain $a$, nor a cofinal subsequence. If $\mu(B)=\emptyset$, then $a \notin B$, so $a \notin A \cup B$, a contradiction. If $\mu(B)=\{a\}$, we are done. If $y \in \mu(B)$, then $y \in B$, but $B$ does not contain a cofinal subsequence, so $A \cup B$ does not either, so $y \in \mu(A \cup B)$, contradiction.

Case 2.3: $\mu(A)=\emptyset: A$ cannot contain a cofinal subsequence. If $\mu(B)=\{a\}$, we are done. $a \in \mu(B)$ does have to hold, so $\mu(B)=\{a, y\}$ is the only remaining possibility. But then $B$ does not contain a cofinal subsequence, and neither does $A \cup B$, so $y \in \mu(A \cup B)$, contradiction.

Case 2.4: $\mu(A)=\{a, y\}: A$ does not contain a cofinal subsequence. If $\mu(B)=\{a\}$, we are done. If $\mu(B)=\emptyset, B$ does not contain a cofinal subsequence (as $a \notin B$ ), so neither does $A \cup B$, so $y \in \mu(A \cup B)$, contradiction. If $y \in \mu(B), B$ does not contain a cofinal subsequence, and we are done again.

Case 3: $\mu(A \cup B)=\{y\}$ : To obtain a contradiction, we need $a \in \mu(A)$ or $a \in \mu(B)$. But in both cases $a \in \mu(A \cup B)$.

Case 4: $\mu(A \cup B)=\emptyset$ : Thus, $A \cup B$ contains no cofinal subsequence. If, e.g. $y \in \mu(A)$, then $y \in \mu(A \cup B)$, if $a \in \mu(A)$, then $a \in \mu(A \cup B)$, so $\mu(A)=\emptyset$.

\section{Example 3.6}

The example show that $(\mu \subseteq)+(\mu P R)+(\mu \|)+(\mu=)+(\mu \cup) \nRightarrow(\mu \in)$. 
Let $U:=\left\{y, x_{i<\omega}\right\}, x_{i}$ a sequence, each $x_{i}$ kills itself, $x_{i}^{1} \succeq x_{i}^{2} \succeq \ldots$ and $y$ is killed by all cofinal subsequences of the $x_{i}$. Then for any $X \subseteq U \mu(X)=\emptyset$ or $\mu(X)=\{y\}$.

$(\mu \subseteq)$ and $(\mu P R)$ hold obviously.

$(\mu \|)$ : Let $A \cup B$ be given. If $y \notin X$, then for all $Y \subseteq X \mu(Y)=\emptyset$. So, if $y \notin A \cup B$, we are done. If $y \in A \cup B$, if $\mu(A \cup B)=\emptyset$, one of $A, B$ must contain a cofinal sequence, it will have $\mu=\emptyset$. If not, then $\mu(A \cup B)=\{y\}$, and this will also hold for the one $y$ is in.

$(\mu=)$ : Let $A \subseteq B, \mu(B) \cap A \neq \emptyset$, show $\mu(A)=\mu(B) \cap A$. But now $\mu(B)=\{y\}, y \in A$, so $B$ does not contain a cofinal subsequence, neither does $\mathrm{A}$, so $\mu(A)=\{y\}$.

$(\mu \cup):(A-\mu(A)) \cap \mu\left(A^{\prime}\right) \neq \emptyset$, so $\mu\left(A^{\prime}\right)=\{y\}$, so $\mu\left(A \cup A^{\prime}\right)=\emptyset$, as $y \in A-\mu(A)$.

But $(\mu \in)$ does not hold: $y \in U-\mu(U)$, but there is no $x$ s.t. $y \notin \mu(\{x, y\})$.

\section{Fact 3.7}

$(\mu w O R)+(\mu \subseteq) \Rightarrow f(X \cup Y) \subseteq f(X) \cup f(Y) \cup(X \cap Y)$

\section{Proof}

$f(X \cup Y) \subseteq f(X) \cup Y, f(X \cup Y) \subseteq X \cup f(Y)$, so $f(X \cup Y) \subseteq(f(X) \cup Y) \cap(X \cup f(Y))$ $=f(X) \cup f(Y) \cup(X \cap Y)$

\section{Proposition 3.8}

The following table is to be read as follows:

Let a logic $\sim$ satisfies $(L L E)$ and $(C C L)$, and define a function $f: \boldsymbol{D}_{\mathcal{L}} \rightarrow \boldsymbol{D}_{\mathcal{L}}$ by $f(M(T)):=M(\overline{\bar{T}})$. Then $f$ is well defined, satisfies $(\mu d p)$, and $\overline{\bar{T}}=T h(f(M(T)))$.

If $\sim$ satisfies a rule in the left hand side, then - provided the additional properties noted in the middle for $\Rightarrow$ hold, too - $f$ will satisfy the property in the right hand side.

Conversely, if $f: \mathcal{Y} \rightarrow \mathcal{P}\left(M_{\mathcal{L}}\right)$ is a function, with $\boldsymbol{D}_{\mathcal{L}} \subseteq \mathcal{Y}$, and we define a logic $\sim$ by $\overline{\bar{T}}:=T h(f(M(T)))$, then $\sim$ satisfies $(L L E)$ and $(C C L)$. If $f$ satisfies $(\mu d p)$, then $f(M(T))=M(\overline{\bar{T}})$.

If $f$ satisfies a property in the right hand side, then - provided the additional properties noted in the middle for $\Leftarrow$ hold, too $-\sim$ will satisfy the property in the left hand side.

If "formula" is noted in the table, this means that, if one of the theories (the one named the same way in Definition 3.3) is equivalent to a formula, we can renounce on $(\mu d p)$. 


\begin{tabular}{|c|c|c|c|}
\hline \multicolumn{4}{|c|}{ Basics } \\
\hline (1.1) & \multirow[t]{2}{*}{$(O R)$} & $\Rightarrow$ & \multirow[t]{2}{*}{$(\mu O R)$} \\
\hline$(1.2)$ & & $\Leftarrow$ & \\
\hline$(2.1)$ & \multirow[t]{2}{*}{$(\operatorname{disjOR})$} & $\Rightarrow$ & \multirow[t]{2}{*}{$(\mu d i s j O R)$} \\
\hline$(2.2)$ & & $\Leftarrow$ & \\
\hline$(3.1)$ & \multirow[t]{2}{*}{$(w O R)$} & $\Rightarrow$ & \multirow[t]{2}{*}{$(\mu w O R)$} \\
\hline$(3.2)$ & & $\Leftarrow$ & \\
\hline$(4.1)$ & \multirow[t]{2}{*}{$(S C)$} & $\Rightarrow$ & \multirow[t]{2}{*}{$(\mu \subseteq)$} \\
\hline$(4.2)$ & & $\Leftarrow$ & \\
\hline$(5.1)$ & \multirow[t]{2}{*}{$(C P)$} & $\Rightarrow$ & \multirow[t]{2}{*}{$(\mu \emptyset)$} \\
\hline$(5.2)$ & & $\Leftarrow$ & \\
\hline$(6.1)$ & \multirow[t]{4}{*}{$(P R)$} & $\Rightarrow$ & \multirow[t]{4}{*}{$(\mu P R)$} \\
\hline$(6.2)$ & & $\Leftarrow(\mu d p)+(\mu \subseteq)$ & \\
\hline$(6.3)$ & & $\notin=$ without $(\mu d p)$ & \\
\hline$(6.4)$ & & $\begin{array}{c}\Leftarrow(\mu \subseteq) \\
T^{\prime} \text { a formula }\end{array}$ & \\
\hline$(6.5)$ & $(P R)$ & $\begin{array}{c}\Leftarrow \\
T^{\prime} \text { a formula }\end{array}$ & $\left(\mu P R^{\prime}\right)$ \\
\hline$(7.1)$ & \multirow[t]{2}{*}{$(C U T)$} & $\Rightarrow$ & \multirow[t]{2}{*}{$\overline{(\mu C U T)}$} \\
\hline$(7.2)$ & & $\Leftarrow$ & \\
\hline \multicolumn{4}{|c|}{ Cumulativity } \\
\hline$\overline{(8.1)}$ & \multirow[t]{2}{*}{$\overline{(C M)}$} & $\Rightarrow$ & \multirow[t]{2}{*}{$\overline{(\mu C M)}$} \\
\hline$(8.2)$ & & $\Leftarrow$ & \\
\hline$(9.1)$ & \multirow[t]{2}{*}{$(\operatorname{Res} M)$} & $\Rightarrow$ & \multirow[t]{2}{*}{$(\mu \operatorname{Res} M)$} \\
\hline$(9.2)$ & & $\Leftarrow$ & \\
\hline$(10.1)$ & \multirow[t]{2}{*}{$(\subseteq \supseteq)$} & $\Rightarrow$ & \multirow[t]{2}{*}{$(\mu \subseteq \supseteq)$} \\
\hline$(10.2)$ & & $\Leftarrow$ & \\
\hline$(11.1)$ & \multirow[t]{2}{*}{$(C U M)$} & $\Rightarrow$ & $(\mu C U M)$ \\
\hline$(11.2)$ & & $\Leftarrow$ & \\
\hline & & Rationality & \\
\hline$(12.1)$ & $($ RatM) & $\Rightarrow$ & $(\mu \operatorname{RatM})$ \\
\hline$(12.2)$ & & $\Leftarrow(\mu d p)$ & \\
\hline$(12.3)$ & & $\notin$ without $(\mu d p)$ & \\
\hline$(12.4)$ & & $\begin{array}{c}\Leftarrow \\
T \text { a formula }\end{array}$ & \\
\hline$(13.1)$ & $(\operatorname{Rat} M=)$ & $\Rightarrow$ & $(\mu=)$ \\
\hline$(13.2)$ & & $\Leftarrow(\mu d p)$ & \\
\hline$(13.3)$ & & $\notin=$ without $(\mu d p)$ & \\
\hline$(13.4)$ & & $\begin{array}{c}\Leftarrow \\
T \text { a formula }\end{array}$ & \\
\hline (14.1) & $\left(\log =^{\prime}\right)$ & $\Rightarrow$ & $\left(\mu=^{\prime}\right)$ \\
\hline$(14.2)$ & & $\Leftarrow(\mu d p)$ & \\
\hline$(14.3)$ & & $\notin=$ without $(\mu d p)$ & \\
\hline$(14.4)$ & & $\Leftarrow T$ a formula & \\
\hline$(15.1)$ & $\overline{(\log \|)}$ & $\Rightarrow$ & $(\mu \|)$ \\
\hline$(15.2)$ & & $\Leftarrow$ & 21 \\
\hline (16.1) & $(\log \cup)$ & $\Rightarrow(\mu \subseteq)+(\mu=)$ & $(\mu \cup)$ \\
\hline$(16.2)$ & & $\Leftarrow(\mu d p)$ & \\
\hline$(16.3)$ & & $\notin$ without $(\mu d p)$ & \\
\hline$(17.1)$ & $\left(\log \cup^{\prime}\right)$ & $\Rightarrow(\mu \subseteq)+(\mu=)$ & $\left(\mu \cup^{\prime}\right)$ \\
\hline$(17.2)$ & & $\Leftarrow(\mu d p)$ & \\
\hline$(17.3)$ & & $\nLeftarrow$ without $(\mu d p)$ & \\
\hline
\end{tabular}




\section{Proof}

Set $f(T):=f(M(T))$, note that $f\left(T \cup T^{\prime}\right):=f\left(M\left(T \cup T^{\prime}\right)\right)=f\left(M(T) \cap M\left(T^{\prime}\right)\right)$.

We show first the general framework.

Let $\sim$ satisfy $(L L E)$ and $(C C L)$. Let $f: \boldsymbol{D}_{\mathcal{L}} \rightarrow \boldsymbol{D}_{\mathcal{L}}$ be defined by $f(M(T)):=M(\overline{\bar{T}})$. If $M(T)=M\left(T^{\prime}\right)$, then $\bar{T}=\overline{T^{\prime}}$, so by $(L L E) \overline{\bar{T}}=\overline{\overline{T^{\prime}}}$, so $f(M(T))=f\left(M\left(T^{\prime}\right)\right)$, so $f$ is well defined and satisfies $(\mu d p)$. By $(C C L) T h(M(\overline{\bar{T}}))=\overline{\bar{T}}$.

Let $f$ be given, and $\sim$ be defined by $\overline{\bar{T}}:=T h(f(M(T)))$. Obviously, $\sim$ satisfies $(L L E)$ and $(C C L)$ (and thus $(R W)$ ). If $f$ satisfies $(\mu d p)$, then $f(M(T))=M\left(T^{\prime}\right)$ for some $T^{\prime}$, and $f(M(T))=M(T h(f(M(T))))=M(\overline{\bar{T}})$ by Fact 3.4. (We will use Fact 3.4 now without further mentioning.)

Next we show the following fact:

(a) If $f$ satisfies $(\mu d p)$, or $T^{\prime}$ is equivalent to a formula, then $T h\left(f(T) \cap M\left(T^{\prime}\right)\right)=\overline{\bar{T}} \cup T^{\prime}$. Case 1, $f$ satisfies $(\mu d p)$. Th $\left(f(M(T)) \cap M\left(T^{\prime}\right)\right)=T h\left(M(\overline{\bar{T}}) \cap M\left(T^{\prime}\right)=\overline{\bar{T}} \cup T^{\prime}\right.$ by Fact $3.3(5)$.

Case 2, $T^{\prime}$ is equivalent to $\phi^{\prime} . T h\left(f(M(T)) \cap M\left(\phi^{\prime}\right)\right)=\overline{T h(f(M(T))) \cup\left\{\phi^{\prime}\right\}}=\overline{\bar{T} \cup\left\{\phi^{\prime}\right\}}$ by Fact $3.3(3)$.

We now prove the individual properties.

(1.1) $(O R) \Rightarrow(\mu O R)$

Let $X=M(T), Y=M\left(T^{\prime}\right) . f(X \cup Y)=f\left(M(T) \cup M\left(T^{\prime}\right)\right)=f\left(M\left(T \vee T^{\prime}\right)\right):=M\left(\overline{\overline{T \vee T^{\prime}}}\right)$ $\subseteq_{(O R)} M\left(\overline{\bar{T}} \cap \overline{\overline{T^{\prime}}}\right)={ }_{(C C L)} M(\overline{\bar{T}}) \cup M\left(\overline{\overline{T^{\prime}}}\right)=: f(X) \cup f(Y)$.

$(1.2)(\mu O R) \Rightarrow(O R)$

$\overline{\overline{T \vee T^{\prime}}}:=T h\left(f\left(M\left(T \vee T^{\prime}\right)\right)\right)=T h\left(f\left(M(T) \cup M\left(T^{\prime}\right)\right)\right) \supseteq{ }_{(\mu O R)} T h\left(f(M(T)) \cup f\left(M\left(T^{\prime}\right)\right)\right)$ $=\left(\right.$ by Fact 3.2) $T h(f(M(T))) \cap T h\left(f\left(M\left(T^{\prime}\right)\right)\right)=: \overline{\bar{T}} \cap \overline{\overline{T^{\prime}}}$.

(2) By $\neg \operatorname{Con}\left(T, T^{\prime}\right) \Leftrightarrow M(T) \cap M\left(T^{\prime}\right)=\emptyset$, we can use directly the proofs for 1 .

(3.1) $(w O R) \Rightarrow(\mu w O R)$

Let $X=M(T), Y=M\left(T^{\prime}\right) . f(X \cup Y)=f\left(M(T) \cup M\left(T^{\prime}\right)\right)=f\left(M\left(T \vee T^{\prime}\right)\right):=M\left(\overline{\overline{T \vee T^{\prime}}}\right)$ $\subseteq_{(w O R)} M\left(\overline{\bar{T}} \cap \overline{T^{\prime}}\right)={ }_{(C C L)} M(\overline{\bar{T}}) \cup M\left(\overline{T^{\prime}}\right)=: f(X) \cup Y$.

$(3.2)(\mu w O R) \Rightarrow(w O R)$

$\overline{\overline{T \vee T^{\prime}}}:=T h\left(f\left(M\left(T \vee T^{\prime}\right)\right)\right)=T h\left(f\left(M(T) \cup M\left(T^{\prime}\right)\right)\right) \supseteq{ }_{(\mu w O R)} T h\left(f(M(T)) \cup M\left(T^{\prime}\right)\right)=$ (by Fact 3.2) $T h(f(M(T))) \cap T h\left(M\left(T^{\prime}\right)\right)=: \overline{\bar{T}} \cap \overline{T^{\prime}}$.

(4.1) $(S C) \Rightarrow(\mu \subseteq)$ 
Trivial.

$(4.2)(\mu \subseteq) \Rightarrow(S C)$

Trivial.

(5.1) $(C P) \Rightarrow(\mu \emptyset)$

Trivial.

$(5.2)(\mu \emptyset) \Rightarrow(C P)$

Trivial.

(6.1) $(P R) \Rightarrow(\mu P R)$ :

Suppose $X:=M(T), Y:=M\left(T^{\prime}\right), X \subseteq Y$, we have to show $f(Y) \cap X \subseteq f(X)$. By prerequisite, $\overline{T^{\prime}} \subseteq \bar{T}$, so $\overline{T \cup T^{\prime}}=\bar{T}$, so $\overline{\overline{T \cup T^{\prime}}}=\overline{\bar{T}}$ by $(L L E)$. By $(P R) \overline{\overline{T \cup T^{\prime}}} \subseteq \overline{\overline{T^{\prime}}} \cup T$, so $f(Y) \cap X=f\left(T^{\prime}\right) \cap M(T)=M\left(\overline{\overline{T^{\prime}}} \cup T\right) \subseteq M\left(\overline{\overline{T \cup T^{\prime}}}\right)=M(\overline{\bar{T}})=f(X)$.

$(6.2)(\mu P R)+(\mu d p)+(\mu \subseteq) \Rightarrow(P R):$

$f(T) \cap M\left(T^{\prime}\right)={ }_{(\mu \subseteq)} f(T) \cap M(T) \cap M\left(T^{\prime}\right)=f(T) \cap M\left(T \cup T^{\prime}\right) \subseteq_{(\mu P R)} f\left(T \cup T^{\prime}\right)$, so $\overline{\overline{T \cup T^{\prime}}}=T h\left(f\left(T \cup T^{\prime}\right)\right) \subseteq T h\left(f(T) \cap M\left(T^{\prime}\right)\right)=\overline{\bar{T} \cup T^{\prime}}$ by (a) above and $(\mu d p)$.

(6.3) $(\mu P R) \nRightarrow(P R)$ without $(\mu d p)$ :

$(\mu P R)$ holds in all preferential structures (see Definition 4.1) by Fact 4.1. Example 4.2 shows that $(D P)$ may fail in the resulting logic.

(6.4) $(\mu P R)+(\mu \subseteq) \Rightarrow(P R)$ if $T^{\prime}$ is classically equivalent to a formula:

It was shown in the proof of (6.2) that $f(T) \cap M\left(\phi^{\prime}\right) \subseteq f\left(T \cup\left\{\phi^{\prime}\right\}\right)$, so $\overline{\overline{T \cup\left\{\phi^{\prime}\right\}}}=$ $T h\left(f\left(T \cup\left\{\phi^{\prime}\right\}\right)\right) \subseteq T h\left(f(T) \cap M\left(\phi^{\prime}\right)\right)=\overline{\bar{T} \cup\left\{\phi^{\prime}\right\}}$ by (a) above.

(6.5) $\left(\mu P R^{\prime}\right) \Rightarrow(P R)$, if $T^{\prime}$ is classically equivalent to a formula:

$f(M(T)) \cap M\left(\phi^{\prime}\right) \subseteq\left(\mu P R^{\prime}\right) f\left(M(T) \cap M\left(\phi^{\prime}\right)\right)=f\left(M\left(T \cup\left\{\phi^{\prime}\right\}\right)\right)$. So again $\overline{\overline{T \cup\left\{\phi^{\prime}\right\}}}=$ $T h\left(f\left(T \cup\left\{\phi^{\prime}\right\}\right)\right) \subseteq T h\left(f(T) \cap M\left(\phi^{\prime}\right)\right)=\overline{\bar{T}} \cup\left\{\phi^{\prime}\right\}$ by (a) above.

(7.1) $(C U T) \Rightarrow(\mu C U T)$

So let $X=M(T), Y=M\left(T^{\prime}\right)$, and $f(T):=M(\overline{\bar{T}}) \subseteq M\left(T^{\prime}\right) \subseteq M(T) \rightarrow \bar{T} \subseteq \overline{T^{\prime}} \subseteq$ $\overline{\bar{T}}={ }_{(L L E)} \overline{\overline{\overline{(\bar{T}}})} \rightarrow($ by $(C U T)) \overline{\bar{T}}=\overline{\overline{(\bar{T})}} \supseteq \overline{\overline{\left(\overline{T^{\prime}}\right)}}=\overline{\overline{T^{\prime}}} \rightarrow f(T)=M(\overline{\bar{T}}) \subseteq M\left(\overline{\overline{T^{\prime}}}\right)=f\left(T^{\prime}\right)$, thus $f(X) \subseteq f(Y)$.

(7.2) $(\mu C U T) \Rightarrow(C U T)$

Let $T \subseteq \overline{T^{\prime}} \subseteq \overline{\bar{T}}$. Thus $f(T) \subseteq M(\overline{\bar{T}}) \subseteq M\left(T^{\prime}\right) \subseteq M(T)$, so by $(\mu C U T) f(T) \subseteq f\left(T^{\prime}\right)$, so $\overline{\bar{T}}=T h(f(T)) \supseteq T h\left(f\left(T^{\prime}\right)\right)=\overline{\overline{T^{\prime}}}$.

(8.1) $(C M) \Rightarrow(\mu C M)$ 
So let $X=M(T), Y=M\left(T^{\prime}\right)$, and $f(T):=M(\overline{\bar{T}}) \subseteq M\left(T^{\prime}\right) \subseteq M(T) \rightarrow \bar{T} \subseteq \overline{T^{\prime}} \subseteq$ $\overline{\bar{T}}={ }_{(L L E)} \overline{\overline{\overline{(\bar{T})}}} \rightarrow($ by $(L L E),(C M)) \overline{\bar{T}}=\overline{\overline{(\bar{T})}} \subseteq \overline{\overline{\left(\overline{T^{\prime}}\right)}}=\overline{\overline{T^{\prime}}} \rightarrow f(T)=M(\overline{\bar{T}}) \supseteq M\left(\overline{\overline{T^{\prime}}}\right)=$ $f\left(T^{\prime}\right)$, thus $f(X) \supseteq f(Y)$.

(8.2) $(\mu C M) \Rightarrow(C M)$

Let $T \subseteq \overline{T^{\prime}} \subseteq \overline{\bar{T}}$. Thus by $(\mu C M)$ and $f(T) \subseteq M(\overline{\bar{T}}) \subseteq M\left(T^{\prime}\right) \subseteq M(T)$, so $f(T) \supseteq f\left(T^{\prime}\right)$ by $(\mu C M)$, so $\overline{\bar{T}}=T h(f(T)) \subseteq T h\left(f\left(T^{\prime}\right)\right)=\overline{\overline{T^{\prime}}}$.

(9.1) $(\operatorname{Res} M) \Rightarrow(\mu \operatorname{Res} M)$

Let $f(X):=M(\overline{\bar{\Delta}}), A:=M(\alpha), B:=M(\beta)$. So $f(X) \subseteq A \cap B \Rightarrow \Delta \sim \alpha, \beta \Rightarrow{ }_{(\operatorname{Res} M)}$ $\Delta, \alpha \sim \beta \Rightarrow M(\overline{\overline{\Delta, \alpha}}) \subseteq M(\beta) \Rightarrow f(X \cap A) \subseteq B$.

(9.2) $(\mu \operatorname{Res} M) \Rightarrow(\operatorname{Res} M)$

Let $f(X):=M(\overline{\bar{\Delta}}), A:=M(\alpha), B:=M(\beta)$. So $\Delta \sim \alpha, \beta \Rightarrow f(X) \subseteq A \cap B \Rightarrow_{(\mu \operatorname{Res} M)}$ $f(X \cap A) \subseteq B \Rightarrow \Delta, \alpha \sim \beta$.

$(10.1)(\subseteq \supseteq) \Rightarrow(\mu \subseteq \supseteq)$

Let $f(T) \subseteq M\left(T^{\prime}\right), f\left(T^{\prime}\right) \subseteq M(T)$. So $T h\left(M\left(T^{\prime}\right)\right) \subseteq T h(f(T)), T h(M(T)) \subseteq T h\left(f\left(T^{\prime}\right)\right)$, so $T^{\prime} \subseteq \overline{T^{\prime}} \subseteq \overline{\bar{T}}, T \subseteq \bar{T} \subseteq \overline{\overline{T^{\prime}}}$, so by $(\subseteq \supseteq) \overline{\bar{T}}=\overline{\overline{T^{\prime}}}$, so $f(T):=M(\overline{\bar{T}})=M\left(\overline{\overline{T^{\prime}}}\right)=: f\left(T^{\prime}\right)$.

$(10.2)(\mu \subseteq \supseteq) \Rightarrow(\subseteq \supseteq)$

Let $T \subseteq \overline{\overline{T^{\prime}}}$ and $T^{\prime} \subseteq \overline{\bar{T}}$. So by $(C C L) T h(M(T))=\bar{T} \subseteq \overline{\overline{T^{\prime}}}=T h\left(f\left(T^{\prime}\right)\right)$. But $T h(M(T)) \subseteq T h(X) \Rightarrow X \subseteq M(T): X \subseteq M(T h(X)) \subseteq M(T h(M(T)))=M(T)$. So $f\left(T^{\prime}\right) \subseteq M(T)$, likewise $f(T) \subseteq M\left(T^{\prime}\right)$, so by $(\mu \subseteq \supseteq) f(T)=f\left(T^{\prime}\right)$, so $\overline{\bar{T}}=\overline{\overline{T^{\prime}}}$.

(11.1) $(C U M) \Rightarrow(\mu C U M)$ :

So let $X=M(T), Y=M\left(T^{\prime}\right)$, and $f(T):=M(\overline{\bar{T}}) \subseteq M\left(T^{\prime}\right) \subseteq M(T) \rightarrow \bar{T} \subseteq \overline{T^{\prime}} \subseteq$ $\overline{\bar{T}}={ }_{(L L E)} \overline{\overline{(\bar{T})}} \rightarrow \overline{\bar{T}}=\overline{\overline{(\bar{T})}}=\overline{\overline{\left(\overline{T^{\prime}}\right)}}=\overline{\overline{T^{\prime}}} \rightarrow f(T)=M(\overline{\bar{T}})=M\left(\overline{\overline{T^{\prime}}}\right)=f\left(T^{\prime}\right)$, thus $f(X)=f(Y)$.

(11.2) $(\mu C U M) \Rightarrow(C U M)$ :

Let $T \subseteq \overline{T^{\prime}} \subseteq \overline{\bar{T}}$. Thus by $(\mu C U M)$ and $f(T) \subseteq M(\overline{\bar{T}}) \subseteq M\left(T^{\prime}\right) \subseteq M(T)$, so $f(T)=f\left(T^{\prime}\right)$, so $\overline{\bar{T}}=T h(f(T))=T h\left(f\left(T^{\prime}\right)\right)=\overline{\overline{T^{\prime}}}$.

(12.1) $(\operatorname{Rat} M) \Rightarrow(\mu \operatorname{Rat} M)$

Let $X=M(T), Y=M\left(T^{\prime}\right)$, and $X \subseteq Y, X \cap f(Y) \neq \emptyset$, so $T \vdash T^{\prime}$ and $M(T) \cap f\left(M\left(T^{\prime}\right)\right) \neq$ $\emptyset$, so $\operatorname{Con}\left(T, \overline{\overline{T^{\prime}}}\right)$, so $\overline{\overline{T^{\prime}}} \cup T \subseteq \overline{\bar{T}}$ by $(\operatorname{Rat} M)$, so $f(X)=f(M(T))=M(\overline{\bar{T}}) \subseteq M\left(\overline{\overline{T^{\prime}}} \cup T\right)=$ $M\left(\overline{\overline{T^{\prime}}}\right) \cap M(T)=f(Y) \cap X$.

(12.2) $(\mu \operatorname{Rat} M)+(\mu d p) \Rightarrow(\operatorname{Rat} M):$

Let $X=M(T), Y=M\left(T^{\prime}\right), T \vdash T^{\prime}, C o n\left(T, \overline{\overline{T^{\prime}}}\right)$, so $X \subseteq Y$ and by $(\mu d p) X \cap f(Y) \neq \emptyset$, so 
by $(\mu R a t M) f(X) \subseteq f(Y) \cap X$, so $\overline{\overline{T \cup T^{\prime}}}=T h\left(f\left(T \cup T^{\prime}\right)\right) \subseteq T h\left(f(T) \cap M\left(T^{\prime}\right)\right)=\overline{\bar{T} \cup T^{\prime}}$ by (a) above and $(\mu d p)$.

(12.3) $(\mu$ Rat $M) \nRightarrow($ Rat $M)$ without $(\mu d p)$ :

$(\mu$ Rat $M)$ holds in all ranked preferential structures (see Definition 4.4) by Fact 4.11. Example 3.7 (2) shows that (RatM) may fail in the resulting logic.

(12.4) $(\mu$ Rat $M) \Rightarrow($ Rat $M)$ if $T$ is classically equivalent to a formula:

$\phi \vdash T^{\prime} \Rightarrow M(\phi) \subseteq M\left(T^{\prime}\right) . \operatorname{Con}\left(\phi, \overline{\overline{T^{\prime}}}\right) \Leftrightarrow M\left(\overline{\overline{T^{\prime}}}\right) \cap M(\phi) \neq \emptyset \Leftrightarrow f\left(T^{\prime}\right) \cap M(\phi)=\emptyset$ by Fact 3.3 (4). Thus $f(M(\phi)) \subseteq f\left(M\left(T^{\prime}\right)\right) \cap M(\phi)$ by $(\mu \operatorname{Rat} M)$. Thus by (a) above $\overline{\overline{\overline{T^{\prime}}} \cup\{\phi\}} \subseteq \overline{\bar{\phi}}$.

(13.1) $($ Rat $M=) \Rightarrow(\mu=)$

Let $X=M(T), Y=M\left(T^{\prime}\right)$, and $X \subseteq Y, X \cap f(Y) \neq \emptyset$, so $T \vdash T^{\prime}$ and $M(T) \cap f\left(M\left(T^{\prime}\right)\right) \neq$ $\emptyset$, so $\operatorname{Con}\left(T, \overline{\overline{T^{\prime}}}\right)$, so $\overline{\overline{T^{\prime}} \cup T}=\overline{\bar{T}}$ by $(\operatorname{Rat} M=)$, so $f(X)=f(M(T))=M(\overline{\bar{T}})=M\left(\overline{\overline{T^{\prime}}} \cup\right.$ $T)=M\left(\overline{\overline{T^{\prime}}}\right) \cap M(T)=f(Y) \cap X$.

$(13.2)(\mu=)+(\mu d p) \Rightarrow(\operatorname{Rat} M=)$

Let $X=M(T), Y=M\left(T^{\prime}\right), T \vdash T^{\prime}, C o n\left(T, \overline{\overline{T^{\prime}}}\right)$, so $X \subseteq Y$ and by $(\mu d p) X \cap f(Y) \neq \emptyset$, so by $(\mu=) f(X)=f(Y) \cap X$. So $\overline{\overline{T^{\prime}} \cup T}=\overline{\bar{T}}$ (a) above and $(\mu d p)$.

(13.3) $(\mu=) \nRightarrow(\operatorname{Rat} M=)$ without $(\mu d p)$ :

$(\mu=)$ holds in all ranked preferential structures (see Definition 4.4) by Fact 4.11. Example 3.7 (1) shows that $(\operatorname{Rat} M=)$ may fail in the resulting logic.

(13.4) $(\mu=) \Rightarrow($ Rat $M=)$ if $T$ is classically equivalent to a formula:

The proof is almost identical to the one for (12.4). Again, the prerequisites of $(\mu=)$ are satisfied, so $f(M(\phi))=f\left(M\left(T^{\prime}\right)\right) \cap M(\phi)$. Thus, $\overline{\overline{\overline{T^{\prime}}} \cup\{\phi\}}=\overline{\bar{\phi}}$ by (a) above.

Of the last four, we show (14), (15), (17), the proof for (16) is similar to the one for (17). (14.1) $\left(\log =^{\prime}\right) \Rightarrow\left(\mu=^{\prime}\right)$ :

$f\left(M\left(T^{\prime}\right)\right) \cap M(T) \neq \emptyset \Rightarrow \operatorname{Con}\left(\overline{\overline{T^{\prime}}} \cup T\right) \Rightarrow_{\left(L_{o g=}\right)} \overline{\overline{T \cup T^{\prime}}}=\overline{\overline{\overline{T^{\prime}}} \cup T} \Rightarrow f\left(M\left(T \cup T^{\prime}\right)\right)=$ $f\left(M\left(T^{\prime}\right)\right) \cap M(T)$.

(14.2) $\left(\mu=^{\prime}\right)+(\mu d p) \Rightarrow\left(\log =^{\prime}\right)$ :

$\operatorname{Con}\left(\overline{\overline{T^{\prime}}} \cup T\right) \Rightarrow_{(\mu d p)} f\left(M\left(T^{\prime}\right)\right) \cap M(T) \neq \emptyset \Rightarrow f\left(M\left(T^{\prime} \cup T\right)\right)=f\left(M\left(T^{\prime}\right) \cap M(T)\right)={ }_{\left(\mu=^{\prime}\right)}$ $f\left(M\left(T^{\prime}\right)\right) \cap M(T)$, so $\overline{\overline{T^{\prime} \cup T}}=\overline{\overline{\overline{T^{\prime}}} \cup T}$ by (a) above and $(\mu d p)$.

(14.3) $\left(\mu=^{\prime}\right) \nRightarrow\left(\log =^{\prime}\right)$ without $(\mu d p)$ :

By Fact $4.11\left(\mu=^{\prime}\right)$ holds in ranked structures. Consider Example $3.7(2)$. There, $\operatorname{Con}\left(T, \overline{\overline{T^{\prime}}}\right), T=T \cup T^{\prime}$, and it was shown that $\overline{\overline{T^{\prime}} \cup T} \nsubseteq \overline{\bar{T}}=\overline{\overline{T \cup T^{\prime}}}$ 
(14.4) $\left(\mu=^{\prime}\right) \Rightarrow\left(\log =^{\prime}\right)$ if $T$ is classically equivalent to a formula:

$\operatorname{Con}\left(\overline{\overline{T^{\prime}}} \cup\{\phi\}\right) \Rightarrow \emptyset \neq M\left(\overline{\overline{T^{\prime}}}\right) \cap M(\phi) \Rightarrow f\left(T^{\prime}\right) \cap M(\phi) \neq \emptyset$ by Fact 3.3 (4). So $f\left(M\left(T^{\prime} \cup\right.\right.$

$\{\phi\}))=f\left(M\left(T^{\prime}\right) \cap M(\phi)\right)=f\left(M\left(T^{\prime}\right)\right) \cap M(\phi)$ by $\left(\mu=^{\prime}\right)$, so $\overline{\overline{T^{\prime} \cup\{\phi\}}}=\overline{\overline{T^{\prime}} \cup\{\phi\}}$ by (a) above.

(15.1) $(\log \|) \Rightarrow(\mu \|)$ :

Trivial.

(15.2) $(\mu \|) \Rightarrow(\log \|):$

Trivial.

(16) $(\log \cup) \Leftrightarrow(\mu \cup)$ : Analogous to the proof of (17).

(17.1) $\left(\log \cup^{\prime}\right)+(\mu \subseteq)+(\mu=) \Rightarrow\left(\mu \cup^{\prime}\right):$

$f\left(M\left(T^{\prime}\right)\right) \cap(M(T)-f(M(T))) \neq \emptyset \Rightarrow\left(\right.$ by $(\mu \subseteq),(\mu=)$, Fact 4.8) $f\left(M\left(T^{\prime}\right)\right) \cap M(T) \neq \emptyset$, $f\left(M\left(T^{\prime}\right)\right) \cap f(M(T))=\emptyset \Rightarrow \operatorname{Con}\left(\overline{\overline{T^{\prime}}}, T\right), \neg \operatorname{Con}\left(\overline{\overline{T^{\prime}}}, \overline{\bar{T}}\right) \Rightarrow \overline{\overline{T \vee T^{\prime}}}=\overline{\bar{T}} \Rightarrow f(M(T))=$ $f\left(M\left(T \vee T^{\prime}\right)\right)=f\left(M(T) \cup M\left(T^{\prime}\right)\right)$.

$(17.2)\left(\mu \cup^{\prime}\right)+(\mu d p) \Rightarrow\left(\log \cup^{\prime}\right):$

$\operatorname{Con}\left(\overline{\overline{T^{\prime}}} \cup T\right), \neg \operatorname{Con}\left(\overline{\overline{T^{\prime}}} \cup \overline{\bar{T}}\right) \Rightarrow_{(\mu d p)} f\left(T^{\prime}\right) \cap M(T) \neq \emptyset, f\left(T^{\prime}\right) \cap f(T)=\emptyset \Rightarrow f\left(M\left(T^{\prime}\right)\right) \cap$ $(M(T)-f(M(T))) \neq \emptyset \Rightarrow f(M(T))=f\left(M(T) \cup M\left(T^{\prime}\right)\right)=f\left(M\left(T \vee T^{\prime}\right)\right)$. So $\overline{\bar{T}}=\overline{\overline{T \vee T^{\prime}}}$.

(17.3) and (16.3) are solved by Example 3.7 (3).

\section{Example 3.7}

(1) $(\mu=)$ without $(\mu d p)$ does not imply $(\operatorname{Rat} M=)$ :

Take $\left\{p_{i}: i \in \omega\right\}$ and put $m:=m_{\bigwedge p_{i}}$, the model which makes all $p_{i}$ true, in the top layer, all the other in the bottom layer. Let $m^{\prime} \neq m, T^{\prime}:=\emptyset, T:=T h\left(m, m^{\prime}\right)$. Then Then $\overline{\overline{T^{\prime}}}=T^{\prime}$, so $\operatorname{Con}\left(\overline{\overline{T^{\prime}}}, T\right), \overline{\bar{T}}=T h\left(m^{\prime}\right), \overline{\overline{T^{\prime}} \cup T}=T$.

So $(\operatorname{Rat} M=)$ fails, but $(\mu=)$ holds in all ranked structures.

(2) $(\mu$ Rat $M)$ without $(\mu d p)$ does not imply (RatM):

Take $\left\{p_{i}: i \in \omega\right\}$ and let $m:=m_{\bigwedge p_{i}}$, the model which makes all $p_{i}$ true.

Let $X:=M\left(\neg p_{0}\right) \cup\{m\}$ be the top layer, put the rest of $M_{\mathcal{L}}$ in the bottom layer. Let $Y:=M_{\mathcal{L}}$. The structure is ranked, as shown in Fact 4.11, $(\mu$ RatM) holds.

Let $T^{\prime}:=\emptyset, T:=T h(X)$. We have to show that $\operatorname{Con}\left(T, \overline{\overline{T^{\prime}}}\right), T \vdash T^{\prime}$, but $\overline{\overline{T^{\prime}} \cup T} \nsubseteq \overline{\bar{T}} . \overline{\overline{T^{\prime}}}$ $=T h\left(M\left(p_{0}\right)-\{m\}\right)=\overline{p_{0}} . T=\overline{\left\{\neg p_{0}\right\} \vee T h(m)}, \overline{\bar{T}}=T$. So $\operatorname{Con}\left(T, \overline{\overline{T^{\prime}}}\right) \cdot M\left(\overline{\overline{T^{\prime}}}\right)=M\left(p_{0}\right)$, 
$M(T)=X, M\left(\overline{\overline{T^{\prime}}} \cup T\right)=M\left(\overline{\overline{T^{\prime}}}\right) \cap M(T)=\{m\}, m \models p_{1}$, so $p_{1} \in \overline{\overline{T^{\prime}}} \cup T$, but $X \not \models p_{1}$.

(3) This example shows that we need $(\mu d p)$ to go from $(\mu \cup)$ to $(\log \cup)$ and from $\left(\mu \cup^{\prime}\right)$ to $\left(\log \cup^{\prime}\right)$.

Let $v(\mathcal{L}):=\{p, q\} \cup\left\{p_{i}: i<\omega\right\}$. Let $m$ make all variables true.

Put all models of $\neg p$, and $m$, in the upper layer, all other models in the lower layer. This is ranked, so by Fact $4.11(\mu \cup)$ and $\left(\mu \cup^{\prime}\right)$ hold. Set $X:=M(\neg q) \cup\{m\}, X^{\prime}:=M(q)-\{m\}$, $T:=T h(X)=\neg q \vee T h(m), T^{\prime}:=T h\left(X^{\prime}\right)=\bar{q}$. Then $\overline{\bar{T}}=\overline{p \wedge \neg q}, \overline{\overline{T^{\prime}}}=\overline{p \wedge q}$. We have $\operatorname{Con}\left(\overline{\overline{T^{\prime}}}, T\right), \neg \operatorname{Con}\left(\overline{\overline{T^{\prime}}}, \overline{\bar{T}}\right)$. But $\overline{\overline{T \vee T^{\prime}}}=\bar{p} \neq \overline{\bar{T}}=\overline{p \wedge \neg q}$ and $\operatorname{Con}\left(\overline{\overline{T \vee T^{\prime}}}, T^{\prime}\right)$, so $(\log \cup)$ and $\left(\log \cup^{\prime}\right)$ fail.

\section{Fact 3.9}

$(C U T) \nRightarrow(P R)$

\section{Proof}

We give two proofs:

(1) If $(C U T) \Rightarrow(P R)$, then by $(\mu P R) \Rightarrow$ (by Fact $3.6(3))(\mu C U T) \Rightarrow$ (by Proposition $3.8(7.2)(C U T) \Rightarrow(P R)$ we would have a proof of $(\mu P R) \Rightarrow(P R)$ without $(\mu d p)$, which is impossible, as shown by Example 4.2.

(2) Reconsider Example 3.3, and say $a \models p \wedge q, b \models p \wedge \neg q, c \models \neg p \wedge q$. It is shown there that $(\mu C U M)$ holds, so $(\mu C U T)$ holds, so by Proposition 3.8 (7.2) (CUT) holds, if we define $\overline{\bar{T}}:=T h\left(f(M(T))\right.$. Set $T:=\{p \vee(\neg p \wedge q)\}, T^{\prime}:=\{p\}$, then $\overline{\overline{T \cup T^{\prime}}}=\overline{\overline{T^{\prime}}}=\overline{\{p \wedge \neg q\}}$, $\overline{\bar{T}}=\bar{T}, \overline{T \cup T^{\prime}}=\overline{T^{\prime}}=\overline{\{p\}}$, so (PR) fails. 


\section{$4 \quad$ Preferential structures}

\subsection{General and smooth preferential structures}

\subsubsection{Definitions and basics}

\section{Definition 4.1}

Fix $U \neq \emptyset$, and consider arbitrary $X$. Note that this $X$ has not necessarily anything to do with $U$, or $\mathcal{U}$ below. Thus, the functions $\mu_{\mathcal{M}}$ below are in principle functions from $V$ to $V$ - where $V$ is the set theoretical universe we work in.

(A) Preferential models or structures.

(1) The version without copies:

A pair $\mathcal{M}:=<U, \prec>$ with $U$ an arbitrary set, and $\prec$ an arbitrary binary relation is called a preferential model or structure.

(2) The version with copies:

A pair $\mathcal{M}:=<\mathcal{U}, \prec>$ with $\mathcal{U}$ an arbitrary set of pairs, and $\prec$ an arbitrary binary relation is called a preferential model or structure.

If $\langle x, i>\in \mathcal{U}$, then $x$ is intended to be an element of $U$, and $i$ the index of the copy.

We sometimes also need copies of the relation $\prec$, we will then replace $\prec$ by one or several arrows $\alpha$ attacking non-minimal elements, e.g. $x \prec y$ will be written $\alpha: x \rightarrow y,<$ $x, i>\prec<y, i>$ will be written $\alpha:\langle x, i>\rightarrow<y, i>$, and finally we might have $\langle\alpha, k>$ : $x \rightarrow y$ and $\langle\alpha, k>:<x, i>\rightarrow<y, i>$, etc.

(B) Minimal elements, the functions $\mu_{\mathcal{M}}$

(1) The version without copies:

Let $\mathcal{M}:=<U, \prec>$, and define

$\mu_{\mathcal{M}}(X):=\left\{x \in X: x \in U \wedge \neg \exists x^{\prime} \in X \cap U . x^{\prime} \prec x\right\}$.

$\mu_{\mathcal{M}}(X)$ is called the set of minimal elements of $X$ (in $\left.\mathcal{M}\right)$.

(2) The version with copies:

Let $\mathcal{M}:=<\mathcal{U}, \prec>$ be as above. Define

$\mu_{\mathcal{M}}(X):=\left\{x \in X: \exists<x, i>\in \mathcal{U} . \neg \exists<x^{\prime}, i^{\prime}>\in \mathcal{U}\left(x^{\prime} \in X \wedge<x^{\prime}, i^{\prime}>^{\prime} \prec<x, i>\right)\right\}$.

Again, by abuse of language, we say that $\mu_{\mathcal{M}}(X)$ is the set of minimal elements of $X$ in the structure. If the context is clear, we will also write just $\mu$.

We sometimes say that $\langle x, i>$ "kills" or "minimizes" $<y, j>$ if $<x, i>\prec<y, j>$. By abuse of language we also say a set $X$ kills or minimizes a set $Y$ if for all $<y, j>\in \mathcal{U}$, 
$y \in Y$ there is $\langle x, i>\in \mathcal{U}, x \in X$ s.t. $\langle x, i>\prec<y, j>$.

$\mathcal{M}$ is also called injective or 1-copy, iff there is always at most one copy $\langle x, i\rangle$ for each $x$. Note that the existence of copies corresponds to a non-injective labelling function - as is often used in nonclassical logic, e.g. modal logic.

We say that $\mathcal{M}$ is transitive, irreflexive, etc., iff $\prec$ is.

Note that $\mu(X)$ might well be empty, even if $X$ is not.

\section{Definition 4.2}

We define the consequence relation of a preferential structure for a given propositional language $\mathcal{L}$.

(A)

(1) If $m$ is a classical model of a language $\mathcal{L}$, we say by abuse of language

$<m, i>\models \phi$ iff $m \models \phi$,

and if $X$ is a set of such pairs, that

$X \models \phi$ iff for all $<m, i>\in X m \models \phi$.

(2) If $\mathcal{M}$ is a preferential structure, and $X$ is a set of $\mathcal{L}$-models for a classical propositional language $\mathcal{L}$, or a set of pairs $\langle m, i\rangle$, where the $m$ are such models, we call $\mathcal{M}$ a classical preferential structure or model.

(B)

Validity in a preferential structure, or the semantical consequence relation defined by such a structure:

Let $\mathcal{M}$ be as above.

We define:

$T \models_{\mathcal{M}} \phi$ iff $\mu_{\mathcal{M}}(M(T)) \models \phi$, i.e. $\mu_{\mathcal{M}}(M(T)) \subseteq M(\phi)$.

$\mathcal{M}$ will be called definability preserving iff for all $X \in \boldsymbol{D}_{\mathcal{L}} \mu_{\mathcal{M}}(X) \in \boldsymbol{D}_{\mathcal{L}}$.

As $\mu_{\mathcal{M}}$ is defined on $\boldsymbol{D}_{\mathcal{L}}$, but need by no means always result in some new definable set, this is (and reveals itself as a quite strong) additional property.

\section{Example 4.1}

This simple example illustrates the importance of copies. Such examples seem to have appeared for the first time in print in [KLM90], but can probably be attibuted to folklore.

Consider the propositional language $\mathcal{L}$ of two propositional variables $p, q$, and the classical preferential model $\mathcal{M}$ defined by 
$m \models p \wedge q, m^{\prime} \models p \wedge q, m_{2} \models \neg p \wedge q, m_{3} \models \neg p \wedge \neg q$, with $m_{2} \prec m, m_{3} \prec m^{\prime}$, and let $\models_{\mathcal{M}}$ be its consequence relation. ( $\mathrm{m}$ and $m^{\prime}$ are logically identical.)

Obviously, $T h(m) \vee\{\neg p\} \models_{\mathcal{M}} \neg p$, but there is no complete theory $T^{\prime}$ s.t. $T h(m) \vee T^{\prime} \models_{\mathcal{M}}$ $\neg p$. (If there were one, $T^{\prime}$ would correspond to $m, m_{2}, m_{3}$, or the missing $m_{4} \models p \wedge \neg q$, but we need two models to kill all copies of $m$.) On the other hand, if there were just one copy of $m$, then one other model, i.e. a complete theory would suffice. More formally, if we admit at most one copy of each model in a structure $\mathcal{M}, m \not \models T$, and $T h(m) \vee T \models_{\mathcal{M}} \phi$ for some $\phi$ s.t. $m \models \neg \phi$ - i.e. $m$ is not minimal in the models of $T h(m) \vee T$ - then there is a complete $T^{\prime}$ with $T^{\prime} \vdash T$ and $T h(m) \vee T^{\prime} \models_{\mathcal{M}} \phi$, i.e. there is $m^{\prime \prime}$ with $m^{\prime \prime} \models T^{\prime}$ and $m^{\prime \prime} \prec m$.

\section{Definition 4.3}

Let $\mathcal{Y} \subseteq \mathcal{P}(U)$. (In applications to logic, $\mathcal{Y}$ will be $\boldsymbol{D}_{\mathcal{L}}$.)

A preferential structure $\mathcal{M}$ is called $\mathcal{Y}$-smooth iff in every $X \in \mathcal{Y}$ every element $x \in X$ is either minimal in $X$ or above an element, which is minimal in $X$. More precisely:

(1) The version without copies:

If $x \in X \in \mathcal{Y}$, then either $x \in \mu(X)$ or there is $x^{\prime} \in \mu(X) \cdot x^{\prime} \prec x$.

(2) The version with copies:

If $x \in X \in \mathcal{Y}$, and $<x, i>\in \mathcal{U}$, then either there is no $<x^{\prime}, i^{\prime}>\in \mathcal{U}, x^{\prime} \in X,<x^{\prime}, i^{\prime}>\prec<$ $x, i>$ or there is $\left\langle x^{\prime}, i^{\prime}>\in \mathcal{U},\left\langle x^{\prime}, i^{\prime}>\prec<x, i>, x^{\prime} \in X\right.\right.$, s.t. there is no $<x^{\prime \prime}, i^{\prime \prime}>\in \mathcal{U}$, $x^{\prime \prime} \in X$, with $<x^{\prime \prime}, i^{\prime \prime}>\prec<x^{\prime}, i^{\prime}>$.

When considering the models of a language $\mathcal{L}, \mathcal{M}$ will be called smooth iff it is $\boldsymbol{D}_{\mathcal{L}}$-smooth; $\boldsymbol{D}_{\mathcal{L}}$ is the default.

Obviously, the richer the set $\mathcal{Y}$ is, the stronger the condition $\mathcal{Y}$-smoothness will be. 


\subsubsection{Representation}

The following table summarizes representation by general or smooth preferential structures. The implications on the right are shown in Proposition 3.8 (going via the $\mu$-functions), those on the left are shown in the respective representation theorems.

\begin{tabular}{|c|c|c|c|c|}
\hline$\mu-$ function & & Pref.Structure & & Logic \\
\hline \multirow[t]{3}{*}{$(\mu \subseteq)+(\mu P R)$} & $\begin{array}{c}\Leftarrow \\
\text { Fact } 4.1\end{array}$ & \multirow[t]{3}{*}{ general } & $\Rightarrow(\mu d p)$ & \multirow[t]{3}{*}{$(L L E)+(R W)+(S C)+(P R)$} \\
\hline & $\begin{array}{c}\Rightarrow \\
\text { Proposition } 4.3\end{array}$ & & $\Leftarrow$ & \\
\hline & & & $\begin{array}{c}\Rightarrow \text { without }(\mu d p) \\
\text { Example } 4.2\end{array}$ & \\
\hline \multirow[t]{3}{*}{$(\mu \subseteq)+(\mu P R)$} & $\begin{array}{c}\Leftarrow \\
\text { Fact } 4.1\end{array}$ & \multirow[t]{3}{*}{ transitive } & $\Rightarrow(\mu d p)$ & \multirow[t]{3}{*}{$(L L E)+(R W)+(S C)+(P R)$} \\
\hline & $\begin{array}{c}\Rightarrow \\
\text { Proposition } 4.4\end{array}$ & & $\Leftarrow$ & \\
\hline & & & $\begin{array}{c}\Rightarrow \text { without }(\mu d p) \\
\text { Example } 4.2\end{array}$ & \\
\hline \multirow[t]{3}{*}{$(\mu \subseteq)+(\mu P R)+(\mu C U M)$} & $\begin{array}{c}\Leftarrow \\
\text { Fact } 4.2\end{array}$ & \multirow[t]{3}{*}{ smooth } & $\Rightarrow(\mu d p)$ & \multirow[t]{3}{*}{$\begin{array}{c}(L L E)+(R W)+(S C)+(P R)+ \\
(C U M)\end{array}$} \\
\hline & $\begin{array}{c}\Rightarrow(\cup) \\
\text { Proposition } 4.5\end{array}$ & & $\Leftarrow(\cup)$ & \\
\hline & & & $\begin{array}{c}\not \Rightarrow \text { without }(\mu d p) \\
\text { Example } 4.2\end{array}$ & \\
\hline \multirow[t]{3}{*}{$(\mu \subseteq)+(\mu P R)+(\mu C U M)$} & $\begin{array}{c}\Leftarrow \\
\text { Fact } 4.2\end{array}$ & \multirow[t]{3}{*}{ smooth+transitive } & $\Rightarrow(\mu d p)$ & \multirow[t]{3}{*}{$\begin{array}{c}(L L E)+(R W)+(S C)+(P R)+ \\
(C U M)\end{array}$} \\
\hline & $\begin{array}{c}\Rightarrow(\cup) \\
\text { Proposition } 4.6\end{array}$ & & $\Leftarrow(\cup)$ & \\
\hline & & & $\begin{array}{c}\nRightarrow \text { without }(\mu d p) \\
\text { Example } 4.2\end{array}$ & \\
\hline
\end{tabular}

\section{Fact 4.1}

$(\mu \subseteq)$ and $(\mu P R)$ hold in all preferential structures.

\section{Proof}

Trivial. The central argument is: if $x, y \in X \subseteq Y$, and $x \prec y$ in $X$, then also $x \prec y$ in $Y$.

\section{Fact 4.2}

$(\mu \subseteq),(\mu P R)$, and $(\mu C U M)$ hold in all smooth preferential structures.

\section{Proof}

By Fact 4.1, we only have to show $(\mu C U M)$. By Fact 3.6, $(\mu C U T)$ follows from $(\mu P R)$, so it remains to show $(\mu C M)$. So suppose $\mu(X) \subseteq Y \subseteq X$, we have to show $\mu(Y) \subseteq \mu(X)$. 
Let $x \in X-\mu(X)$, so there is $x^{\prime} \in X, x^{\prime} \prec x$, by smoothness, there must be $x^{\prime \prime} \in \mu(X)$, $x^{\prime \prime} \prec x$, so $x^{\prime \prime} \in Y$, and $x \notin \mu(Y)$. The proof for the case with copies is analogous.

\section{Example 4.2}

This example was first given in [Sch92]. It shows that condition $(P R)$ may fail in preferential structures which are not definability preserving.

Let $v(\mathcal{L}):=\left\{p_{i}: i \in \omega\right\}, n, n^{\prime} \in M_{\mathcal{L}}$ be defined by $n \models\left\{p_{i}: i \in \omega\right\}, n^{\prime} \models\left\{\neg p_{0}\right\} \cup\left\{p_{i}:\right.$ $0<i<\omega\}$.

Let $\mathcal{M}:=<M_{\mathcal{L}}, \prec>$ where only $n \prec n^{\prime}$, i.e. just two models are comparable. Note that the structure is transitive and smooth. Thus, by Fact $4.2(\mu \subseteq),(\mu P R),(\mu C U M)$ hold.

Let $\mu:=\mu_{\mathcal{M}}$, and $\sim$ be defined as usual by $\mu$.

Set $T:=\emptyset, T^{\prime}:=\left\{p_{i}: 0<i<\omega\right\}$. We have $M_{T}=M_{\mathcal{L}}, f\left(M_{T}\right)=M_{\mathcal{L}}-\left\{n^{\prime}\right\}$, $M_{T^{\prime}}=\left\{n, n^{\prime}\right\}, f\left(M_{T^{\prime}}\right)=\{n\}$. So by the result of Example 3.1, $f$ is not definability preserving, and, furthermore, $\overline{\bar{T}}=\bar{T}, \overline{\overline{T^{\prime}}}=\overline{\left\{p_{i}: i<\omega\right\}}$, so $p_{0} \in \overline{\overline{T \cup T^{\prime}}}$, but $\overline{\bar{T} \cup T^{\prime}}=\overline{\bar{T} \cup T^{\prime}}=\overline{T^{\prime}}$, so $p_{0} \notin \overline{\bar{T} \cup T^{\prime}}$, contradicting $(P R)$, which holds in all definability preserving preferential structures

\section{Proposition 4.3}

Let $\mu: \mathcal{Y} \rightarrow \mathcal{P}(U)$ satisfy $(\mu \subseteq)$ and $(\mu P R)$. Then there is a preferential structure $\mathcal{X}$ s.t. $\mu=\mu_{\mathcal{X}}$.

\section{Proposition 4.4}

Let $\mu: \mathcal{Y} \rightarrow \mathcal{P}(U)$ satisfy $(\mu \subseteq)$ and $(\mu P R)$. Then there is a transitive preferential structure $\mathcal{X}$ s.t. $\mu=\mu_{\mathcal{X}}$.

\section{Proposition 4.5}

Let $\mu: \mathcal{Y} \rightarrow \mathcal{P}(U)$ satisfy $(\mu \subseteq),(\mu P R)$, and $(\mu C U M)$, and the domain $\mathcal{Y}(\cup)$.

Then there is a $\mathcal{Y}$-smooth preferential structure $\mathcal{X}$ s.t. $\mu=\mu_{\mathcal{X}}$.

\section{Proposition 4.6}

Let $\mu: \mathcal{Y} \rightarrow \mathcal{P}(U)$ satisfy $(\mu \subseteq),(\mu P R)$, and $(\mu C U M)$, and the domain $\mathcal{Y}(\cup)$.

Then there is a transitive $\mathcal{Y}$-smooth preferential structure $\mathcal{X}$ s.t. $\mu=\mu_{\mathcal{X}}$. 


\subsection{Ranked structures}

\subsubsection{Definitions and basics}

\section{Fact 4.7}

Let $\prec$ be an irreflexive, binary relation on $X$, then the following two conditions are equivalent:

(1) There is $\Omega$ and an irreflexive, total, binary relation $\prec^{\prime}$ on $\Omega$ and a function $f: X \rightarrow \Omega$ s.t. $x \prec y \leftrightarrow f(x) \prec^{\prime} f(y)$ for all $x, y \in X$.

(2) Let $x, y, z \in X$ and $x \perp y$ wrt. $\prec$ (i.e. neither $x \prec y$ nor $y \prec x$ ), then $z \prec x \rightarrow z \prec y$ and $x \prec z \rightarrow y \prec z$.

\section{Definition 4.4}

We call an irreflexive, binary relation $\prec$ on $X$, which satisfies (1) (equivalently (2)) of Fact 4.7 , ranked. By abuse of language, we also call a preferential structure $\langle X, \prec>$ ranked, iff $\prec$ is.

\section{Fact 4.8}

$M(T)-M\left(T^{\prime}\right)$ is normally not definable.

In the presence of $(\mu=)$ and $(\mu \subseteq), f(Y) \cap(X-f(X)) \neq \emptyset$ is equivalent to $f(Y) \cap X \neq \emptyset$ and $f(Y) \cap f(X)=\emptyset$.

\section{Proof}

$f(Y) \cap(X-f(X))=(f(Y) \cap X)-(f(Y) \cap f(X))$.

" $\Leftarrow "$ : Let $f(Y) \cap X \neq \emptyset, f(Y) \cap f(X)=\emptyset$, so $f(Y) \cap(X-f(X)) \neq \emptyset$.

" $\Rightarrow$ ": Suppose $f(Y) \cap(X-f(X)) \neq \emptyset$, so $f(Y) \cap X \neq \emptyset$. Suppose $f(Y) \cap f(X) \neq \emptyset$, so by $(\mu \subseteq) f(Y) \cap X \cap Y \neq \emptyset$, so by $(\mu=) f(Y) \cap X \cap Y=f(X \cap Y)$, and $f(X) \cap X \cap Y \neq \emptyset$, so by $(\mu=) f(X) \cap X \cap Y=f(X \cap Y)$, so $f(X) \cap Y=f(Y) \cap X$ and $f(Y) \cap(X-f(X))=\emptyset$.

\section{Fact 4.9}

If $\prec$ on $X$ is ranked, and free of cycles, then $\prec$ is transitive. 


\section{Proof}

Let $x \prec y \prec z$. If $x \perp z$, then $y \succ z$, resulting in a cycle of length 2 . If $z \prec x$, then we have a cycle of length 3 . So $x \prec z$.

\section{Remark 4.10}

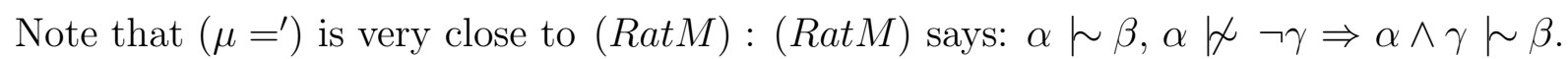
Or, $f(A) \subseteq B, f(A) \cap C \neq \emptyset \rightarrow f(A \cap C) \subseteq B$ for all $A, B, C$. This is not quite, but almost: $f(A \cap C) \subseteq f(A) \cap C$ (it depends how many $B$ there are, if $f(A)$ is some such $B$, the fit is perfect).

\section{Fact 4.11}

In all ranked structures, $(\mu \subseteq),(\mu=),(\mu P R),\left(\mu=^{\prime}\right),(\mu \|),(\mu \cup),\left(\mu \cup^{\prime}\right),(\mu \in),(\mu R a t M)$ will hold, if the corresponding closure conditions are satisfied.

\section{Proof:}

$(\mu \subseteq)$ and $(\mu P R)$ hold in all preferential structures.

$(\mu=)$ and $\left(\mu=^{\prime}\right)$ are trivial.

$(\mu \cup)$ and $\left(\mu \cup^{\prime}\right)$ : All minimal copies of elements in $f(Y)$ have the same rank. If some $y \in f(Y)$ has all its minimal copies killed by an element $x \in X$, by rankedness, $x$ kills the rest, too.

$(\mu \in):$ If $f(\{a\})=\emptyset$, we are done. Take the minimal copies of a in $\{a\}$, they are all killed by one element in $X$.

$(\mu \|)$ : Case $f(X)=\emptyset$ : If below every copy of $y \in Y$ there is a copy of some $x \in X$, then $f(X \cup Y)=\emptyset$. Otherwise $f(X \cup Y)=f(Y)$. Suppose now $f(X) \neq \emptyset, f(Y) \neq \emptyset$, then the minimal ranks decide: if they are equal, $f(X \cup Y)=f(X) \cup f(Y)$, etc.

$(\mu$ Rat $M):$ Let $X \subseteq Y, y \in X \cap f(Y) \neq \emptyset, x \in f(X)$. By rankedness, $y \prec x$, or $y \perp x$, $y \prec x$ is impossible, as $y \in X$, so $y \perp x$, and $x \in f(Y)$. 


\subsubsection{Representation}

\section{Definition 4.5}

Let $\mathcal{Z}=<\mathcal{X}, \prec>$ be a preferential structure. Call $\mathcal{Z} 1-\infty$ over $Z$, iff for all $x \in Z$ there are exactly one or infinitely many copies of $x$, i.e. for all $x \in Z\{u \in \mathcal{X}: u=<x, i>$ for some $i$ \} has cardinality 1 or $\geq \omega$.

\section{Lemma 4.12}

Let $\mathcal{Z}=<\mathcal{X}, \prec>$ be a preferential structure and $f: \mathcal{Y} \rightarrow \mathcal{P}(Z)$ with $\mathcal{Y} \subseteq \mathcal{P}(Z)$ be represented by $\mathcal{Z}$, i.e. for $X \in \mathcal{Y} f(X)=\mu_{\mathcal{Z}}(X)$, and $\mathcal{Z}$ be ranked and free of cycles. Then there is a structure $\mathcal{Z}^{\prime}, 1-\infty$ over $Z$, ranked and free of cycles, which also represents $f$.

\section{Proof}

We construct $\mathcal{Z}^{\prime}=\left\langle\mathcal{X}^{\prime}, \prec^{\prime}\right\rangle$.

Let $A:=\{x \in Z$ : there is some $\langle x, i>\in \mathcal{X}$, but for all $<x, i>\in \mathcal{X}$ there is $\langle x, j\rangle \in \mathcal{X}$ with $<x, j>\prec<x, i>\}$,

let $B:=\{x \in Z$ : there is some $<x, i>\in \mathcal{X}$, s.t. for no $<x, j>\in \mathcal{X}<x, j>\prec<x, i>\}$, let $C:=\{x \in Z:$ there is no $<x, i>\in \mathcal{X}\}$.

Let $c_{i}: i<\kappa$ be an enumeration of $C$. We introduce for each such $c_{i} \omega$ many copies $<c_{i}, n>: n<\omega$ into $\mathcal{X}^{\prime}$, put all $<c_{i}, n>$ above all elements in $\mathcal{X}$, and order the $<c_{i}, n>$ by $<c_{i}, n>\prec^{\prime}<c_{i^{\prime}}, n^{\prime}>: \leftrightarrow\left(i=i^{\prime}\right.$ and $\left.n>n^{\prime}\right)$ or $i>i^{\prime}$. Thus, all $<c_{i}, n>$ are comparable.

If $a \in A$, then there are infinitely many copies of a in $\mathcal{X}$, as $\mathcal{X}$ was cycle-free, we put them all into $\mathcal{X}^{\prime}$. If $b \in B$, we choose exactly one such minimal element $<b, m>$ (i.e. there is no $<b, n>\prec<b, m>$ ) into $\mathcal{X}^{\prime}$, and omit all other elements. (For definiteness, assume in all applications $m=0$.) For all elements from $\mathrm{A}$ and $B$, we take the restriction of the order $\prec$ of $\mathcal{X}$. This is the new structure $\mathcal{Z}^{\prime}$.

Obviously, adding the $\left\langle c_{i}, n\right\rangle$ does not introduce cycles, irreflexivity and rankedness are preserved. Moreover, any substructure of a cycle-free, irreflexive, ranked structure also has these properties, so $\mathcal{Z}^{\prime}$ is $1-\infty$ over $Z$, ranked and free of cycles.

We show that $\mathcal{Z}$ and $\mathcal{Z}^{\prime}$ are equivalent. Let then $X \subseteq Z$, we have to prove $\mu(X)=\mu^{\prime}(X)$ $\left(\mu:=\mu_{\mathcal{Z}}, \mu^{\prime}:=\mu_{\mathcal{Z}^{\prime}}\right)$.

Let $z \in X-\mu(X)$. If $z \in C$ or $z \in A$, then $z \notin \mu^{\prime}(X)$. If $z \in B$, let $<z, m>$ be the chosen element. As $z \notin \mu(X)$, there is $x \in X$ s.t. some $<x, j>\prec<z, m>$. $x$ cannot be in $C$. If $x \in A$, then also $<x, j>\prec^{\prime}<z, m>$. If $x \in B$, then there is some $\langle x, k>$ also in $\mathcal{X}^{\prime} .<x, j>\prec<x, k>$ is impossible. If $\langle x, k>\prec<x, j>$, then $\langle z, m>\succ<x, k>$ 
by transitivity. If $\langle x, k\rangle \perp<x, j\rangle$, then also $\langle z, m\rangle \succ<x, k>$ by rankedness. In any case, $\langle z, m\rangle \succ^{\prime}\langle x, k\rangle$, and thus $z \notin \mu^{\prime}(X)$.

Let $z \in X-\mu^{\prime}(X)$. If $z \in C$ or $z \in A$, then $z \notin \mu(X)$. Let $z \in B$, and some $<x, j>\prec^{\prime}<z, m>$. $x$ cannot be in $C$, as they were sorted on top, so $\langle x, j\rangle$ exists in $\mathcal{X}$ too and $\langle x, j\rangle \prec<z, m\rangle$. But if any other $\langle z, i>$ is also minimal in $\mathcal{Z}$ among the $\langle z, k>$, then by rankedness also $\langle x, j\rangle \prec<z, i>$, as $\langle z, i>\perp<z, m>$, so $z \notin \mu(X)$.

\section{Proposition 4.13}

The first result applies for structures without copies of elements.

(1) Let $\mathcal{Y} \subseteq \mathcal{P}(U)$ be closed under finite unions. Then $(\mu \subseteq),(\mu \emptyset),(\mu=)$ characterize ranked structures for which for all $X \in \mathcal{Y} X \neq \emptyset \rightarrow \mu_{<}(X) \neq \emptyset$ hold, i.e. $(\mu \subseteq)$, $(\mu \emptyset)$, $(\mu=)$ hold in such structures for $\mu_{<}$, and if they hold for some $\mu$, we can find a ranked relation $<$ on $U$ s.t. $\mu=\mu_{<}$. Moreover, the structure can be choosen $\mathcal{Y}$-smooth.

(2) Let $\mathcal{Y} \subseteq \mathcal{P}(U)$ be closed under finite unions, and contain singletons. Then $(\mu \subseteq)$, $(\mu \emptyset$ fin $),(\mu=),(\mu \in)$ characterize ranked structures for which for all finite $X \in \mathcal{Y} X \neq \emptyset$ $\rightarrow \mu_{<}(X) \neq \emptyset$ hold, i.e. $(\mu \subseteq),(\mu \emptyset$ fin $),(\mu=),(\mu \in)$ hold in such structures for $\mu_{<}$, and if they hold for some $\mu$, we can find a ranked relation $<$ on $U$ s.t. $\mu=\mu_{<}$.

Note that the prerequisites of (2) hold in particular in the case of ranked structures without copies, where all elements of $U$ are present in the structure - we need infinite descending chains to have $\mu(X)=\emptyset$ for $X \neq \emptyset$.

\section{Fact 4.14}

$(\mu \subseteq)+(\mu P R)+(\mu=)+(\mu \cup)+(\mu \in)$ do not imply representation by a ranked structure.

\section{Proof}

See Example 4.3.

\section{Example 4.3}

This example shows that the conditions $(\mu \subseteq)+(\mu P R)+(\mu=)+(\mu \cup)+(\mu \in)$ can be satisfied, and still representation by a ranked structure is impossible.

Consider $\mu(\{a, b\})=\emptyset, \mu(\{a\})=\{a\}, \mu(\{b\})=\{b\}$. The conditions $(\mu \subseteq)+(\mu P R)+(\mu=$ )$+(\mu \cup)+(\mu \in)$ hold trivially. This is representable, e.g. by $a_{1} \succeq b_{1} \succeq a_{2} \succeq b_{2} \ldots$ without 
transitivity. (Note that rankedness implies transitivity, $a \preceq b \preceq c$, but not for $a=c$.) But this cannot be represented by a ranked structure: As $\mu(\{a\}) \neq \emptyset$, there must be a copy $a_{i}$ of minimal rank, likewise for $b$ and some $b_{i}$. If they have the same rank, $\mu(\{a, b\})=\{a, b\}$, otherwise it will be $\{a\}$ or $\{b\}$.

\section{Proposition 4.15}

Let $\mathcal{Y}$ be closed under finite unions and contain singletons. Then $(\mu \subseteq)+(\mu P R)+(\mu \|$ )$+(\mu \cup)+(\mu \in)$ characterize ranked structures, where elements may appear in several copies. 


\section{Theory revision}

\subsection{AGM revision}

All material in this Section 5.1 is due verbatim or in essence to AGM - AGM for Alchourron, Gardenfors, Makinson, see e.g. [AGM85].

\section{Definition 5.1}

We present in parallel the logical and the semantic (or purely algebraic) side. For the latter, we work in some fixed universe $U$, and the intuition is $U=M_{\mathcal{L}}, X=M(K)$, etc., so, e.g. $A \in K$ becomes $X \subseteq B$, etc.

(For reasons of readability, we omit most caveats about definability.)

$K_{\perp}$ will denote the inconsistent theory.

We consider two functions, - and $*$, taking a deductively closed theory and a formula as arguments, and returning a (deductively closed) theory on the logics side. The algebraic counterparts work on definable model sets. It is obvious that $(K-1),(K * 1),(K-6)$, $(K * 6)$ have vacuously true counterparts on the semantical side. Note that $K(X)$ will never change, everything is relative to fixed $K(X) . K * \phi$ is the result of revising $K$ with $\phi . K-\phi$ is the result of subtracting enough from $K$ to be able to add $\neg \phi$ in a reasonable way, called contraction.

Moreover, let $\leq_{K}$ be a relation on the formulas relative to a deductively closed theory $K$ on the formulas of $\mathcal{L}$, and $\leq_{X}$ a relation on $\mathcal{P}(U)$ or a suitable subset of $\mathcal{P}(U)$ relative to fixed $X$. When the context is clear, we simply write $\leq_{.} \leq_{K}\left(\leq_{X}\right)$ is called a relation of epistemic entrenchment for $K(X)$.

The following table presents the "rationality postulates" for contraction (-), revision (*) and epistemic entrenchment. In AGM tradition, $K$ will be a deductively closed theory, $\phi, \psi$ formulas. Accordingly, $X$ will be the set of models of a theory, $A, B$ the model sets of formulas. 


\begin{tabular}{|c|c|c|c|}
\hline \multicolumn{4}{|c|}{ Contraction, $K-\phi$} \\
\hline$(K-1)$ & $K-\phi$ is deductively closed & & \\
\hline$\overline{(K-2)}$ & $K-\phi \subseteq K$ & $(X \ominus 2)$ & $X \subseteq X \ominus A$ \\
\hline$(K-3)$ & $\phi \notin K \Rightarrow K-\phi=K$ & $(X \ominus 3)$ & $X \nsubseteq A \Rightarrow X \ominus A=X$ \\
\hline$(K-4)$ & $\forall \phi \Rightarrow \phi \notin K-\phi$ & $(X \ominus 4)$ & $A \neq U \Rightarrow X \ominus A \nsubseteq A$ \\
\hline$(K-5)$ & $\overline{K \subseteq \overline{(K-\phi) \cup\{\phi\}}}$ & $(X \ominus 5)$ & $(X \ominus A) \cap A \subseteq X$ \\
\hline$(K-6)$ & $\vdash \phi \leftrightarrow \psi \Rightarrow K-\phi=K-\psi$ & & \\
\hline$(K-7)$ & $\begin{array}{c}(K-\phi) \cap(K-\psi) \subseteq \\
K-(\phi \wedge \psi)\end{array}$ & $(X \ominus 7)$ & $\begin{array}{c}X \ominus(A \cap B) \subseteq \\
(X \ominus A) \cup(X \ominus B)\end{array}$ \\
\hline$(K-8)$ & $\begin{array}{c}\phi \notin K-(\phi \wedge \psi) \Rightarrow \\
K-(\phi \wedge \psi) \subseteq K-\phi\end{array}$ & $(X \ominus 8)$ & $\begin{array}{c}X \ominus(A \cap B) \nsubseteq \subseteq A \Rightarrow \\
X \ominus A \subseteq X \ominus(A \cap B)\end{array}$ \\
\hline \multicolumn{4}{|c|}{ Revision, $K * \phi$} \\
\hline$(K * 1)$ & $K * \phi$ is deductively closed & - & \\
\hline$(K * 2)$ & $\phi \in K * \phi$ & $(X \mid 2)$ & $X \mid A \subseteq A$ \\
\hline$(K * 3)$ & $\overline{K * \phi \subseteq \overline{K \cup\{\phi\}}}$ & $(X \mid 3)$ & $X \cap A \subseteq X \mid A$ \\
\hline$(K * 4)$ & 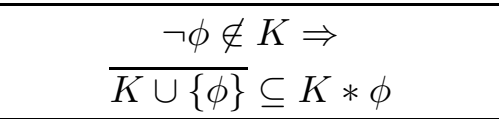 & $(X \mid 4)$ & $\begin{array}{c}X \cap A \neq \emptyset \Rightarrow \\
X \mid A \subseteq X \cap A\end{array}$ \\
\hline$(K * 5)$ & $K * \phi=K_{\perp} \Rightarrow \vdash \neg \phi$ & $(X \mid 5)$ & $X \mid A=\emptyset \Rightarrow A=\emptyset$ \\
\hline$(K * 6)$ & $\vdash \phi \leftrightarrow \psi \Rightarrow K * \phi=K * \psi$ & - & \\
\hline$(K * 7)$ & $\frac{K *(\phi \wedge \psi) \subseteq}{(K * \phi) \cup\{\psi\}}$ & $(X \mid 7)$ & $\begin{array}{c}(X \mid A) \cap B \subseteq \\
X \mid(A \cap B)\end{array}$ \\
\hline$(K * 8)$ & $\begin{array}{c}\neg \psi \notin K * \phi \Rightarrow \\
\overline{(K * \phi) \cup\{\psi\} \subseteq K *(\phi \wedge \psi)}\end{array}$ & $(X \mid 8)$ & $\begin{array}{c}(X \mid A) \cap B \neq \emptyset \Rightarrow \\
X \mid(A \cap B) \subseteq(X \mid A) \cap B\end{array}$ \\
\hline \multicolumn{4}{|c|}{ Epistemic entrenchment } \\
\hline$(E E 1)$ & $\leq_{K}$ is transitive & $(E E 1)$ & $\leq_{X}$ is transitive \\
\hline$(E E 2)$ & $\phi \vdash \psi \Rightarrow \phi \leq_{K} \psi$ & $(E E 2)$ & $A \subseteq B \Rightarrow A \leq_{X} B$ \\
\hline$(E E 3)$ & $\begin{array}{c}\forall \phi, \psi \\
\left(\phi \leq_{K} \phi \wedge \psi \text { or } \psi \leq_{K} \phi \wedge \psi\right)\end{array}$ & $(E E 3)$ & $\begin{array}{c}\forall A, B \\
\left(A \leq_{X} A \cap B \text { or } B \leq_{X} A \cap B\right)\end{array}$ \\
\hline$(E E 4)$ & $\begin{array}{c}K \neq K_{\perp} \Rightarrow \\
\left(\phi \notin K \text { iff } \forall \psi \cdot \phi \leq_{K} \psi\right)\end{array}$ & $(E E 4)$ & $\begin{array}{c}X \neq \emptyset \Rightarrow \\
\left(X \not \subset A \text { iff } \forall B . A \leq_{X} B\right)\end{array}$ \\
\hline$(E E 5)$ & $\forall \psi \cdot \psi \leq_{K} \phi \Rightarrow \vdash \phi$ & $(E E 5)$ & $\forall B . B \leq_{X} A \Rightarrow A=U$ \\
\hline
\end{tabular}

\section{Remark 5.1}

(1) Note that $(X \mid 7)$ and $(X \mid 8)$ express a central condition for ranked structures, see Section 3.10: If we note $X \mid$. by $f_{X}($.$) , we then have: f_{X}(A) \cap B \neq \emptyset \Rightarrow f_{X}(A \cap B)=$ $f_{X}(A) \cap B$. 
(2) It is trivial to see that AGM revision cannot be defined by an individual distance (see Definition 2.3.5 below): Suppose $X \mid Y:=\left\{y \in Y: \exists x_{y} \in X\left(\forall y^{\prime} \in Y . d\left(x_{y}, y\right) \leq\right.\right.$ $\left.\left.d\left(x_{y}, y^{\prime}\right)\right)\right\}$. Consider $a, b, c .\{a, b\} \mid\{b, c\}=\{b\}$ by $(X \mid 3)$ and $(X \mid 4)$, so $d(a, b)<d(a, c)$. But on the other hand $\{a, c\} \mid\{b, c\}=\{c\}$, so $d(a, b)>d(a, c)$, contradiction.

\section{Proposition 5.2}

Contraction, revision, and epistemic entrenchment are interdefinable by the following equations, i.e., if the defining side has the respective properties, so will the defined side.

\begin{tabular}{|c|c|c|c|c|c|}
\hline \multicolumn{2}{|c|}{$K * \phi:=\overline{(K-\neg \phi)} \cup \phi$} & \multicolumn{4}{|c|}{$X \mid A:=(X \ominus \boldsymbol{C A} A) \cap A$} \\
\hline \multicolumn{2}{|c|}{$K-\phi:=K \cap(K * \neg \phi)$} & \multicolumn{4}{|c|}{$X \ominus A:=X \cup(X \mid \boldsymbol{C} A)$} \\
\hline \multicolumn{2}{|c|}{$K-\phi:=\left\{\psi \in K:\left(\phi<_{K} \phi \vee \psi\right.\right.$ or $\left.\left.\vdash \phi\right)\right\}$} & $X \ominus A:=$ & $\bigcap\{B: X \subseteq B \subseteq$ & $\begin{array}{r}X \quad \text { iff } \\
\left.U, A<_{X} A \cup B\right\}\end{array}$ & $\begin{array}{l}A=U, \\
\text { otherwise }\end{array}$ \\
\hline$\phi \leq_{K} \psi: \leftrightarrow\{$ & $\begin{array}{l}\vdash \phi \wedge \psi \\
\text { or } \\
\phi \notin K-(\phi \wedge \psi)\end{array}$ & & $A \leq_{X} B: \leftrightarrow\{$ & $\begin{array}{l}A, B=U \\
\text { or } \\
X \ominus(A \cap B) \nsubseteq A\end{array}$ & \\
\hline
\end{tabular}

The idea of epistemic entrenchment is that $\phi$ is more entrenched than $\psi$ (relative to $K$ ) iff $M(\neg \psi)$ is closer to $M(K)$ than $M(\neg \phi)$ is to $M(K)$. In shorthand, the more we can twiggle $K$ without reaching $\neg \phi$, the more $\phi$ is entrenched. Truth is maximally entrenched - no twiggling whatever will reach falsity. The more $\phi$ is entrenched, the more we are certain about it. Seen this way, the properties of epistemic entrenchment relations are very natural (and trivial): As only the closest points of $M(\neg \phi)$ count (seen from $M(K)$ ), $\phi$ or $\psi$ will be as entrenched as $\phi \wedge \psi$, and there is a logically strongest $\phi^{\prime}$ which is as entrenched as $\phi$ - this is just the sphere around $M(K)$ with radius $d(M(K), M(\neg \phi))$. 


\subsection{Distance based revision}

\subsubsection{Definitions and basics}

\section{Definition 5.2}

$d: U \times U \rightarrow Z$ is called a pseudo-distance on $U$ iff (d1) holds:

(d1) $Z$ is totally ordered by a relation $<$.

If, in addition, $Z$ has a $<-$ smallest element 0 , and (d2) holds, we say that $d$ respects identity:

(d2) $d(a, b)=0$ iff $a=b$.

If, in addition, (d3) holds, then $d$ is called symmetric:

(d3) $d(a, b)=d(b, a)$.

(For any $a, b \in U$.)

Note that we can force the triangle inequality to hold trivially (if we can choose the values in the real numbers): It suffices to choose the values in the set $\{0\} \cup[0.5,1]$, i.e. in the interval from 0.5 to 1 , or as 0 .

\section{Definition 5.3}

We define the collective and the individual variant of choosing the closest elements in the second operand by two operators, $\mid, \uparrow: \mathcal{P}(U) \times \mathcal{P}(U) \rightarrow \mathcal{P}(U)$ :

Let $d$ be a distance or pseudo-distance.

$X \mid Y:=\left\{y \in Y: \exists x_{y} \in X . \forall x^{\prime} \in X, \forall y^{\prime} \in Y\left(d\left(x_{y}, y\right) \leq d\left(x^{\prime}, y^{\prime}\right)\right\}\right.$

(the collective variant, used in theory revision)

and

$X \uparrow Y:=\left\{y \in Y: \exists x_{y} \in X . \forall y^{\prime} \in Y\left(d\left(x_{y}, y\right) \leq d\left(x_{y}, y^{\prime}\right)\right\}\right.$

(the individual variant, used for counterfactual conditionals and theory update).

Thus, $\left.A\right|_{d} B$ is the subset of $B$ consisting of all $b \in B$ that are closest to A. Note that, if $A$ or $B$ is infinite, $\left.A\right|_{d} B$ may be empty, even if $A$ and $B$ are not empty. A condition assuring nonemptiness will be imposed when necessary.

\section{Definition 5.4}

An operation $\mid: \mathcal{P}(U) \times \mathcal{P}(U) \rightarrow \mathcal{P}(U)$ is representable iff there is a pseudo-distance $d: U \times U \rightarrow Z$ such that

$A|B=A|_{d} B:=\left\{b \in B: \exists a_{b} \in A \forall a^{\prime} \in A \forall b^{\prime} \in B\left(d\left(a_{b}, b\right) \leq d\left(a^{\prime}, b^{\prime}\right)\right)\right\}$. 
The following is the central definition, it describes the way a revision $*_{d}$ is attached to a pseudo-distance $d$ on the set of models.

\section{Definition 5.5}

$T *_{d} T^{\prime}:=T h\left(\left.M(T)\right|_{d} M\left(T^{\prime}\right)\right)$.

$*$ is called representable iff there is a pseudo-distance $d$ on the set of models s.t. $T * T^{\prime}=$ $T h\left(\left.M(T)\right|_{d} M\left(T^{\prime}\right)\right)$.

\subsubsection{Representation}

\section{Fact 5.3}

A distance based revision satisfies the AGM postulates provided:

(1) it respects identity, i.e. $d(a, a)<d(a, b)$ for all $a \neq b$,

(2) it satisfies a limit condition: minima exist,

(3) it is definability preserving.

(It is trivial to see that the first two are necessary, and Example 5.1 (2) below shows the necessity of (3). In particular, (2) and (3) will hold for finite languages.)

\section{Proof:}

We use $\mid$ to abbreviate $\left.\right|_{d}$. As a matter of fact, we show slightly more, as we admit also full theories on the right of $*$.

$(K * 1),(K * 2),(K * 6)$ hold by definition, $(K * 3)$ and $(K * 4)$ as $d$ respects identity, $(K * 5)$ by existence of minima.

It remains to show $(K * 7)$ and $(K * 8)$, we do them together, and show: If $T * T^{\prime}$ is consistent with $T^{\prime \prime}$, then $T *\left(T^{\prime} \cup T^{\prime \prime}\right)=\overline{\left(T * T^{\prime}\right) \cup T^{\prime \prime}}$.

Note that $M\left(S \cup S^{\prime}\right)=M(S) \cap M\left(S^{\prime}\right)$, and that $M\left(S * S^{\prime}\right)=M(S) \mid M\left(S^{\prime}\right)$. (The latter is only true if $\mid$ is definability preserving.) By prerequisite, $M\left(T * T^{\prime}\right) \cap M\left(T^{\prime \prime}\right) \neq \emptyset$, so $\left(M(T) \mid M\left(T^{\prime}\right)\right) \cap M\left(T^{\prime \prime}\right) \neq \emptyset$. Let $A:=M(T), B:=M\left(T^{\prime}\right), C:=M\left(T^{\prime \prime}\right)$. " $\subseteq$ ": Let $b \in A \mid(B \cap C)$. By prerequisite, there is $b^{\prime} \in(A \mid B) \cap C$. Thus $d\left(A, b^{\prime}\right) \geq d(A, B \cap C)=$ $d(A, b)$. As $b \in B, b \in A \mid B$, but $b \in C$, too. " $\supseteq$ ": Let $b^{\prime} \in(A \mid B) \cap C$. Thus $d\left(A, b^{\prime}\right)=d(A, B) \leq d(A, B \cap C)$, so by $b^{\prime} \in B \cap C b^{\prime} \in A \mid(B \cap C)$. We conclude $M(T) \mid$ $\left(M\left(T^{\prime}\right) \cap M\left(T^{\prime \prime}\right)\right)=\left(M(T) \mid M\left(T^{\prime}\right)\right) \cap M\left(T^{\prime \prime}\right)$, thus that $T *\left(T^{\prime} \cup T^{\prime \prime}\right)=\overline{\left(T * T^{\prime}\right) \cup T^{\prime \prime}}$. 


\section{Definition 5.6}

For $X, Y \neq \emptyset$, set $U_{Y}(X):=\{z: d(X, z) \leq d(X, Y)\}$.

\section{Fact 5.4}

Let $X, Y, Z \neq \emptyset$. Then

(1) $U_{Y}(X) \cap Z \neq \emptyset$ iff $(X \mid(Y \cup Z)) \cap Z \neq \emptyset$,

(2) $U_{Y}(X) \cap Z \neq \emptyset$ iff $\boldsymbol{C Z} \leq_{X} \boldsymbol{C} Y$ - where $\leq_{X}$ is epistemic entrenchement relative to $X$.

\section{Proof}

(1) Trivial.

(2) $\boldsymbol{C} Z \leq_{X} \boldsymbol{C Y}$ iff $X \ominus(\boldsymbol{C} Z \cap \boldsymbol{C Y}) \not \boldsymbol{C} Z . X \ominus(\boldsymbol{C Z} \cap \boldsymbol{C Y})=X \cup(X \mid \boldsymbol{C}(\boldsymbol{C Z} \cap \boldsymbol{C} Y))$ $=X \cup(X \mid(Z \cup Y))$. So $X \ominus(\boldsymbol{C} Z \cap \boldsymbol{C} Y) \nsubseteq \boldsymbol{C Z} \Leftrightarrow(X \cup(X \mid(Z \cup Y))) \cap Z \neq \emptyset \Leftrightarrow$ $X \cap Z \neq \emptyset$ or $(X \mid(Z \cup Y)) \cap Z \neq \emptyset \Leftrightarrow d(X, Z) \leq d(X, Y)$.

\section{Condition 5.1}

Let $U \neq \emptyset, \mathcal{Y} \subseteq \mathcal{P}(U)$ satisfy $(\cap),(\cup), \emptyset \notin \mathcal{Y}$.

Let $A, B, X_{i} \in \mathcal{Y}, \mid: \mathcal{Y} \times \mathcal{Y} \rightarrow \mathcal{P}(U)$.

Let $*$ be a revision function defined for arbitrary consistent theories on both sides. (This is thus a slight extension of the AGM framework, as AGM work with formulas only on the right of $*$.)

\begin{tabular}{|c|c|c|}
\hline & & 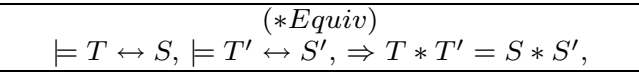 \\
\hline & & $\begin{array}{l}(* C C L) \\
T * T^{\prime} \text { is a consistent, deductively closed theory, }\end{array}$ \\
\hline & $\begin{array}{c}(\mid \text { Succ }) \\
A \mid B \subseteq B\end{array}$ & $\begin{array}{c}(* \text { Succ }) \\
T^{\prime} \subseteq T * T^{\prime} \\
\end{array}$ \\
\hline & $\begin{array}{c}(\mid \text { Con }) \\
A \cap B \neq \emptyset \Rightarrow A \mid B=A \cap B\end{array}$ & 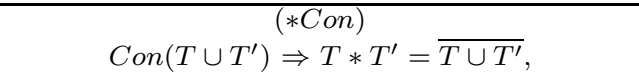 \\
\hline $\begin{array}{c}\text { Intuitively, } \\
\text { Using symmetry } \\
d\left(X_{0}, X_{1}\right) \leq d\left(X_{1}, X_{2}\right) \\
d\left(X_{1}, X_{2}\right) \leq d\left(X_{2}, X_{3}\right), \\
d\left(X_{2}, X_{3}\right) \leq d\left(X_{3}, X_{4}\right) \\
\cdots \\
d\left(X_{k-1}, X_{k}\right) \leq d\left(X_{0}, X_{k}\right) \\
\Rightarrow \\
d\left(X_{0}, X_{1}\right) \leq d\left(X_{0}, X_{k}\right) \\
\text { i.e. transitivity, or absence of } \\
\text { loops involving }<\end{array}$ & $\begin{array}{c}(\mid \text { Loop }) \\
\\
\left(X_{1} \mid\left(X_{0} \cup X_{2}\right)\right) \cap X_{0} \neq \emptyset, \\
\left(X_{2} \mid\left(X_{1} \cup X_{3}\right)\right) \cap X_{1} \neq \emptyset, \\
\left(X_{3} \mid\left(X_{2} \cup X_{4}\right)\right) \cap X_{2} \neq \emptyset, \\
\cdots \\
\left(X_{k} \mid\left(X_{k-1} \cup X_{0}\right)\right) \cap X_{k-1} \neq \emptyset \\
\Rightarrow \\
\left(X_{0} \mid\left(X_{k} \cup X_{1}\right)\right) \cap X_{1} \neq \emptyset\end{array}$ & $\begin{array}{c}(* \text { Loop }) \\
\text { Con }\left(T_{0}, T_{1} *\left(T_{0} \vee T_{2}\right)\right), \\
\operatorname{Con}\left(T_{1}, T_{2} *\left(T_{1} \vee T_{3}\right)\right) \\
\operatorname{Con}\left(T_{2}, T_{3} *\left(T_{2} \vee T_{4}\right)\right) \\
\cdots \\
\operatorname{Con}\left(T_{k-1}, T_{k} *\left(T_{k-1} \vee T_{0}\right)\right) \\
\Rightarrow \\
\operatorname{Con}\left(T_{1}, T_{0} *\left(T_{k} \vee T_{1}\right)\right)\end{array}$ \\
\hline
\end{tabular}




\section{Proposition 5.5}

The following connections between the logical and the algebraic side might be the most interesting ones. We will consider in all cases also the variant with full theories.

Given $*$ which respects logical equivalence, let $M(T) \mid M\left(T^{\prime}\right):=M\left(T * T^{\prime}\right)$, conversely, given |, let $T * T^{\prime}:=T h\left(M(T) \mid M\left(T^{\prime}\right)\right)$. We then have:

\begin{tabular}{|c|c|c|c|}
\hline$(1.1)$ & \multirow[t]{4}{*}{$(K * 7)$} & $\Rightarrow$ & \multirow[t]{4}{*}{$(X \mid 7)$} \\
\hline$(1.2)$ & & $\Leftarrow(\mu d p)$ & \\
\hline$(1.3)$ & & $\Leftarrow \mathrm{B}$ is the model set for some $\phi$ & \\
\hline$(1.4)$ & & $\notin$ in general & \\
\hline$\overline{(2.1)}$ & \multirow[t]{4}{*}{$(*$ Loop $)$} & $\Rightarrow$ & \multirow[t]{4}{*}{$(\mid$ Loop $)$} \\
\hline$(2.2)$ & & $\Leftarrow(\mu d p)$ & \\
\hline$\overline{(2.3)}$ & & $\Leftarrow$ all $X_{i}$ are the model sets for some $\phi_{i}$ & \\
\hline$(2.4)$ & & $\notin$ in general & \\
\hline
\end{tabular}

\section{Proof}

(1)

We consider the equivalence of $T *\left(T^{\prime} \cup T^{\prime \prime}\right) \subseteq \overline{\left(T * T^{\prime}\right) \cup T^{\prime \prime}}$ and $\left(M(T) \mid M\left(T^{\prime}\right)\right) \cap$ $M\left(T^{\prime \prime}\right) \subseteq M(T) \mid\left(M\left(T^{\prime}\right) \cap M\left(T^{\prime \prime}\right)\right)$.

$\left(M(T) \mid M\left(T^{\prime}\right)\right) \cap M\left(T^{\prime \prime}\right)=M\left(T * T^{\prime}\right) \cap M\left(T^{\prime \prime}\right)=M\left(\left(T * T^{\prime}\right) \cup T^{\prime \prime}\right) \subseteq_{(K * 7)} M\left(T *\left(T^{\prime} \cup T^{\prime \prime}\right)\right)$ $=M(T)\left|M\left(T^{\prime} \cup T^{\prime \prime}\right)=M(T)\right|\left(M\left(T^{\prime}\right) \cap M\left(T^{\prime \prime}\right)\right)$.

$T *\left(T^{\prime} \cup T^{\prime \prime}\right)=T h\left(M(T) \mid M\left(T^{\prime} \cup T^{\prime \prime}\right)\right)=T h\left(M(T) \mid\left(M\left(T^{\prime}\right) \cap M\left(T^{\prime \prime}\right)\right)\right) \subseteq_{(X \mid 7)} T h((M(T) \mid$ $\left.\left.\left.M\left(T^{\prime}\right)\right) \cap M\left(T^{\prime \prime}\right)\right)\right)={ }_{(\mu d p)} T h\left(M(T) \mid M\left(T^{\prime}\right)\right) \cup T^{\prime \prime}=T h\left(M\left(\left(T * T^{\prime}\right) \cup T^{\prime \prime}=\overline{\left(T * T^{\prime}\right) \cup T^{\prime \prime}}\right.\right.$.

Let $T^{\prime \prime}$ be equivalent to $\phi^{\prime \prime}$. We can then replace the use of $(\mu d p)$ in the proof of $(1.2)$ by Fact $3.3(3)$.

By Example $5.1(2),(K * 7)$ may fail, though $(X \mid 7)$ holds.

(2.1) and (2.2):

$\operatorname{Con}\left(T_{0}, T_{1} *\left(T_{0} \vee T_{2}\right)\right) \Leftrightarrow M\left(T_{0}\right) \cap M\left(T_{1} *\left(T_{0} \vee T_{2}\right)\right) \neq \emptyset$.

$M\left(T_{1} *\left(T_{0} \vee T_{2}\right)\right)=M\left(T h\left(M\left(T_{1}\right) \mid M\left(T_{0} \vee T_{2}\right)\right)\right)=M\left(T h\left(M\left(T_{1}\right) \mid\left(M\left(T_{0}\right) \cup M\left(T_{2}\right)\right)\right)\right)$ $={ }_{(\mu d p)} M\left(T_{1}\right) \mid\left(M\left(T_{0}\right) \cup\left(T_{2}\right)\right)$, so $\operatorname{Con}\left(T_{0}, T_{1} *\left(T_{0} \vee T_{2}\right)\right) \Leftrightarrow M\left(T_{0}\right) \cap\left(M\left(T_{1}\right) \mid\left(M\left(T_{0}\right) \cup\right.\right.$ $\left.\left.\left(T_{2}\right)\right)\right) \neq \emptyset$.

Thus, all conditions translate one-to-one, and we use (| Loop) and (*Loop) to go back 
and forth.

(2.3):

Let $A:=M\left(T h\left(M\left(T_{1}\right) \mid\left(M\left(T_{0}\right) \cup M\left(T_{2}\right)\right)\right)\right), A^{\prime}:=M\left(T_{1}\right) \mid\left(M\left(T_{0}\right) \cup\left(T_{2}\right)\right)$, then we do not need $A=A^{\prime}$, it suffices to have $M\left(T_{0}\right) \cap A \neq \emptyset \Leftrightarrow M\left(T_{0}\right) \cap A^{\prime} \neq \emptyset . A=\overbrace{A^{\prime}}$, so we can use Fact $3.5(\mathrm{Cl} \cap+)$, if $T_{0}$ is equivalent to some $\phi_{0}$.

This has to hold for all $T_{i}$, so all $T_{i}$ have to be equivalent to some $\phi_{i}$.

By Proposition 5.6, all distance defined | satisfy (| Loop). By Example $5.1(1)$, (*Loop) may fail.

The following Example 5.1 shows that, in general, a revision operation defined on models via a pseudo-distance by $T * T^{\prime}:=T h\left(\left.M(T)\right|_{d} M\left(T^{\prime}\right)\right)$ might not satisfy (*Loop) or $(K * 7)$, unless we require $\left.\right|_{d}$ to preserve definability.

\section{Example 5.1}

Consider an infinite propositional language $\mathcal{L}$.

Let $X$ be an infinite set of models, $m, m_{1}, m_{2}$ be models for $\mathcal{L}$. Arrange the models of $\mathcal{L}$ in the real plane s.t. all $x \in X$ have the same distance $<2$ (in the real plane) from $m$, $m_{2}$ has distance 2 from $m$, and $m_{1}$ has distance 3 from $m$.

Let $T, T_{1}, T_{2}$ be complete (consistent) theories, $T^{\prime}$ a theory with infinitely many models, $M(T)=\{m\}, M\left(T_{1}\right)=\left\{m_{1}\right\}, M\left(T_{2}\right)=\left\{m_{2}\right\}$. The two variants diverge now slightly:

(1) $M\left(T^{\prime}\right)=X \cup\left\{m_{1}\right\}$. T, $T^{\prime}, T_{2}$ will be pairwise inconsistent.

(2) $M\left(T^{\prime}\right)=X \cup\left\{m_{1}, m_{2}\right\}, M\left(T^{\prime \prime}\right)=\left\{m_{1}, m_{2}\right\}$.

Assume in both cases $T h(X)=T^{\prime}$, so $X$ will not be definable by a theory.

Now for the results:

Then $M(T) \mid M\left(T^{\prime}\right)=X$, but $T * T^{\prime}=T h(X)=T^{\prime}$.

(1) We easily verify $\operatorname{Con}\left(T, T_{2} *(T \vee T)\right), \operatorname{Con}\left(T_{2}, T *\left(T_{2} \vee T_{1}\right)\right), \operatorname{Con}\left(T, T_{1} *(T \vee T)\right)$, $\operatorname{Con}\left(T_{1}, T *\left(T_{1} \vee T^{\prime}\right)\right), \operatorname{Con}\left(T, T^{\prime} *(T \vee T)\right)$, and conclude by Loop (i.e. $\left.(* \operatorname{Loop})\right) \operatorname{Con}\left(T_{2}, T *\right.$ $\left.\left(T^{\prime} \vee T_{2}\right)\right)$, which is wrong.

(2) So $T * T^{\prime}$ is consistent with $T^{\prime \prime}$, and $\overline{\left(T * T^{\prime}\right) \cup T^{\prime \prime}}=T^{\prime \prime}$. But $T^{\prime} \cup T^{\prime \prime}=T^{\prime \prime}$, and $T *\left(T^{\prime} \cup T^{\prime \prime}\right)=T_{2} \neq T^{\prime \prime}$, contradicting $(K * 7)$. 


\section{Proposition 5.6}

Let $U \neq \emptyset, \mathcal{Y} \subseteq \mathcal{P}(U)$ be closed under finite $\cap$ and finite $\cup, \emptyset \notin \mathcal{Y}$.

(a) | is representable by a symmetric pseudo-distance $d: U \times U \rightarrow Z$ iff $\mid$ satisfies (| Succ) and $(\mid$ Loop) in Conditions 5.1.

(b) | is representable by an identity respecting symmetric pseudo-distance $d: U \times U \rightarrow Z$ iff | satisfies (|Succ), (|Con), and (| Loop) in Conditions 5.1.

\section{Proposition 5.7}

Let $\mathcal{L}$ be a propositional language.

(a) A revision operation $*$ is representable by a symmetric consistency and definability preserving pseudo-distance iff $*$ satisfies $(*$ Equiv), $(* C C L),(* S u c c),(*$ Loop $)$.

(b) A revision operation $*$ is representable by a symmetric consistency and definability preserving, identity respecting pseudo-distance iff $*$ satisfies $(* E q u i v),(* C C L),(* S u c c)$, $(*$ Con $),(*$ Loop $)$.

\section{Example 5.2}

Note that even when the pseudo-distance is a real distance, the resulting revision operator $\left.\right|_{d}$ does not always permit to reconstruct the relations of the distances: revision is a coarse instrument to investigate distances.

Distances with common start (or end, by symmetry) can always be compared by looking at the result of revision:

$\left.a\right|_{d}\left\{b, b^{\prime}\right\}=b$ iff $d(a, b)<d\left(a, b^{\prime}\right)$,

$\left.a\right|_{d}\left\{b, b^{\prime}\right\}=b^{\prime}$ iff $d(a, b)>d\left(a, b^{\prime}\right)$,

$\left.a\right|_{d}\left\{b, b^{\prime}\right\}=\left\{b, b^{\prime}\right\}$ iff $d(a, b)=d\left(a, b^{\prime}\right)$.

This is not the case with arbitrary distances $d(x, y)$ and $d(a, b)$, as this example will show.

We work in the real plane, with the standard distance, the angles have 120 degrees. $a^{\prime}$ is closer to $y$ than $x$ is to $y$, a is closer to $b$ than $x$ is to $y$, but $a^{\prime}$ is farther away from $b^{\prime}$ than $x$ is from $y$. Similarly for $b, b^{\prime}$. But we cannot distinguish the situation $\{a, b, x, y\}$ and the situation $\left\{a^{\prime}, b^{\prime}, x, y\right\}$ through $\left.\right|_{d}$. (See Diagram 5.1):

Seen from a, the distances are in that order: $y, b, x$.

Seen from $a^{\prime}$, the distances are in that order: $y, b^{\prime}, x$.

Seen from $b$, the distances are in that order: $y, a, x$. 
Seen from $b^{\prime}$, the distances are in that order: $y, a^{\prime}, x$.

Seen from $y$, the distances are in that order: $a / b, x$.

Seen from $y$, the distances are in that order: $a^{\prime} / b^{\prime}, x$.

Seen from $x$, the distances are in that order: $y, a / b$.

Seen from $x$, the distances are in that order: $y, a^{\prime} / b^{\prime}$.

Thus, any $c \mid{ }_{d} C$ will be the same in both situations (with a interchanged with $a^{\prime}, b$ with $\left.b^{\prime}\right)$. The same holds for any $X \mid{ }_{d} C$ where $X$ has two elements.

Thus, any $\left.C\right|_{d} D$ will be the same in both situations, when we interchange a with $a^{\prime}$, and $b$ with $b^{\prime}$. So we cannot determine by $\left.\right|_{d}$ whether $d(x, y)>d(a, b)$ or not.
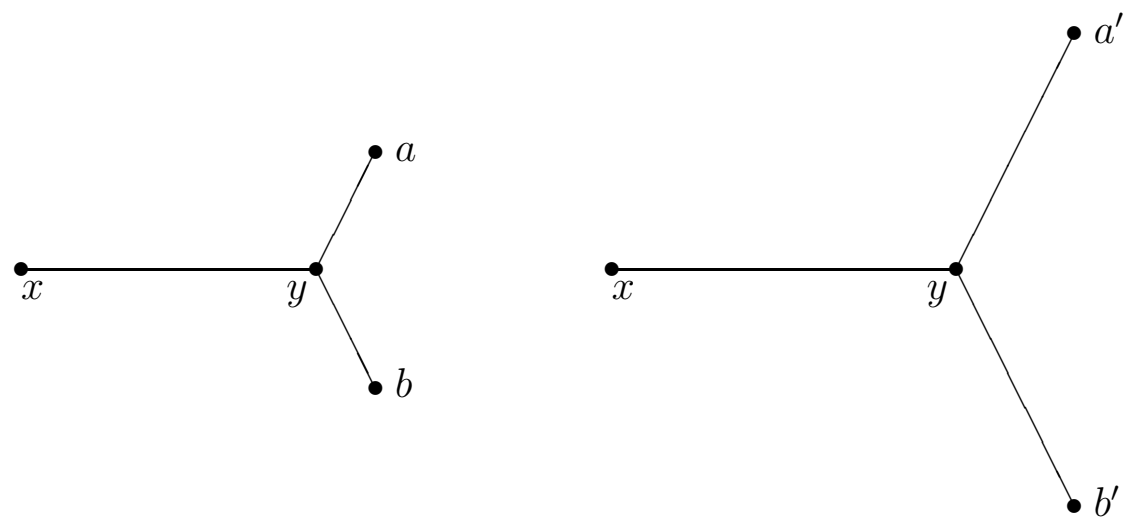

Indiscernible by revision

\section{Diagram 5.1}




\section{Size}

\section{Definition 6.1}

A filter is an abstract notion of size, elements of a filter $\mathcal{F}(X)$ on $X$ are called big subsets of $X$, their complements are called small, and the rest have medium size. The dual applies to ideals $\mathcal{I}(X)$, this is justified by the trivial fact that $\{X-A: A \in \mathcal{F}(X)\}$ is an ideal iff $\mathcal{F}(X)$ is a filter.

In both definitions, the first two conditions (i.e. (FAll), $(I \emptyset)$, and $(F \uparrow),(I \downarrow))$ should hold if the notions shall have anything to do with usual intuition, and there are reasons to consider only the weaker, less idealistic, version of the third.

At the same time, we introduce in rough parallel coherence conditions which describe what might happen when we change the reference or base set $X$. $(R \uparrow)$ is very natural, $(R \downarrow)$ is more daring, and $(R \downarrow \downarrow)$ even more so. $(R \cup d i s j)$ is a cautious combination of $(R \uparrow)$ and $(R \cup)$, as we avoid using the same big set several times in comparison, so $(R \cup)$ is used more cautiously here. See Remark 6.1 for more details.

Finally, we give a generalized first order quantifier corresponding to a (weak) filter. The precise connection is formulated in Definition 6.2, Definition 6.3, Definition 6.4, and Proposition 6.4, respectively their relativized versions.

Fix now a base set $X \neq \emptyset$.

A (weak) filter on or over $X$ is a set $\mathcal{F}(X) \subseteq \mathcal{P}(X)$, s.t. (FAll), $(F \uparrow),(F \cap)((F A l l)$, $(F \uparrow),\left(F \cap^{\prime}\right)$ respectively) hold.

A filter is called a principal filter iff there is $X^{\prime} \subseteq X$ s.t. $\mathcal{F}=\left\{A: X^{\prime} \subseteq A \subseteq X\right\}$.

A filter is called an ultrafilter iff for all $X^{\prime} \subseteq X X^{\prime} \in \mathcal{F}(X)$ or $X-X^{\prime} \in \mathcal{F}(X)$.

A (weak) ideal on or over $X$ is a set $\mathcal{I}(X) \subseteq \mathcal{P}(X)$, s.t. $(I \emptyset),(I \downarrow),(I \cup)((I \emptyset),(I \downarrow)$, $\left(I \cup^{\prime}\right)$ respectively) hold.

Finally, we set $\mathcal{M}(X):=\{A \subseteq X: A \notin \mathcal{I}(X), A \notin \mathcal{F}(X)\}$, the "medium size" sets, and $\mathcal{M}^{+}(X):=\mathcal{M}(X) \cup \mathcal{F}(X), \mathcal{M}^{+}(X)$ is the set of subsets of $X$, which are not small, i.e. have medium or large size.

For $(R \downarrow)$ and $(R \downarrow \downarrow)$ closure under set difference is assumed in the following table. 


\begin{tabular}{|c|c|c|c|}
\hline \multicolumn{4}{|c|}{ Optimum } \\
\hline $\begin{array}{c}(F A l l) \\
X \in \mathcal{F}(X)\end{array}$ & $\begin{array}{c}(I \emptyset) \\
\emptyset \in \mathcal{I}(X)\end{array}$ & & $\forall x \phi(x) \rightarrow \nabla x \phi(x)$ \\
\hline \multicolumn{4}{|c|}{ Improvement } \\
\hline $\begin{array}{c}(F \uparrow) \\
A \subseteq B \subseteq X \\
A \in \mathcal{F}(X) \Rightarrow \\
B \in \mathcal{F}(X)\end{array}$ & $\begin{array}{c}(I \downarrow) \\
A \subseteq B \subseteq X \\
B \in \mathcal{I}(X) \Rightarrow \\
A \in \mathcal{I}(X)\end{array}$ & $\begin{array}{c}(R \uparrow) \\
X \subseteq Y \Rightarrow \mathcal{I}(X) \subseteq \mathcal{I}(Y)\end{array}$ & $\begin{array}{c}\nabla x \phi(x) \wedge \\
\forall x(\phi(x) \rightarrow \psi(x)) \rightarrow \\
\nabla x \psi(x)\end{array}$ \\
\hline \multicolumn{4}{|c|}{ Adding small sets } \\
\hline $\begin{array}{c}(F \cap) \\
A, B \in \mathcal{F}(X) \Rightarrow \\
A \cap B \in \mathcal{F}(X)\end{array}$ & $\begin{array}{c}(I \cup) \\
A, B \in \mathcal{I}(X) \Rightarrow \\
A \cup B \in \mathcal{I}(X)\end{array}$ & $\begin{array}{c}(R \downarrow) \\
A, B \in \mathcal{I}(X) \Rightarrow \\
A-B \in \mathcal{I}(X-\mathrm{B}) \\
\text { or: } \\
A \in \mathcal{F}(X), B \in \mathcal{I}(X) \Rightarrow \\
A-B \in \mathcal{F}(X-\mathrm{B})\end{array}$ & $\begin{array}{c}\nabla x \phi(x) \wedge \nabla x \psi(x) \rightarrow \\
\nabla x(\phi(x) \wedge \psi(x))\end{array}$ \\
\hline \multicolumn{4}{|c|}{ Cautious addition } \\
\hline $\begin{array}{c}\left(F \cap^{\prime}\right) \\
A, B \in \mathcal{F}(X) \Rightarrow \\
A \cap B \neq \emptyset .\end{array}$ & $\begin{array}{c}\left(I \cup^{\prime}\right) \\
A, B \in \mathcal{I}(X) \Rightarrow \\
A \cup B \neq X\end{array}$ & $\begin{array}{c}(R \cup \text { disj }) \\
A \in \mathcal{I}(X), B \in \mathcal{I}(Y), X \cap Y=\emptyset \Rightarrow \\
A \cup B \in \mathcal{I}(X \cup Y)\end{array}$ & $\begin{array}{r}\nabla x \phi(x) \rightarrow \neg \nabla x \neg \phi(x) \\
\text { and } \nabla x \phi(x) \rightarrow \exists x \phi(x)\end{array}$ \\
\hline \multicolumn{4}{|c|}{ Bold addition } \\
\hline Ultrafilter & (Dual of) Ultrafilter & $\begin{array}{c}(R \downarrow \downarrow) \\
A \in \mathcal{I}(X), B \notin \mathcal{F}(X) \Rightarrow \\
A-B \in \mathcal{I}(X-B) \\
\text { or: } \\
A \in \mathcal{F}(X), B \notin \mathcal{F}(X) \Rightarrow \\
A-B \in \mathcal{F}(X-\mathrm{B}) \\
\text { or: } \\
A \in \mathcal{M}^{+}(X), X \in \mathcal{M}^{+}(Y) \Rightarrow \\
A \in \mathcal{M}^{+}(Y)-\text { Transitivity of } \mathcal{M}^{+}\end{array}$ & $\neg \nabla x \phi(x) \rightarrow \nabla x \neg \phi(x)$ \\
\hline
\end{tabular}

These notions are related to nonmonotonic logics as follows:

We can say that, normally, $\phi$ implies $\psi$ iff in a big subset of all $\phi$-cases, $\psi$ holds. In preferential terms, $\phi$ implies $\psi$ iff $\psi$ holds in all minimal $\phi$-models. If $\mu$ is the model choice function of a preferential structure, i.e. $\mu(\phi)$ is the set of minimal $\phi$-models, then $\mu(\phi)$ will be a (the smallest) big subset of the set of $\phi$-models, and the filter over the $\phi$-models is the pricipal filter generated by $\mu(\phi)$.

Due to the finite intersection property, filters and ideals work well with logics: If $\phi$ holds normally, as it holds in a big subset, and so does $\phi^{\prime}$, then $\phi \wedge \phi^{\prime}$ will normally hold, too, as the intersection of two big subsets is big again. This is a nice property, but not justified in all situations, consider e.g. simple counting of a finite subset. (The question has a name, "lottery paradox": normally no single participant wins, but someone wins in the end.) This motivates the weak versions.

Normality defined by (weak or not) filters is a local concept: the filter defined on $X$ and the one defined on $X^{\prime}$ might be totally independent.

Seen more abstractly, set properties like e.g. $(R \uparrow)$ allow the transfer of big (or small) 
subsets from one to another base set (and the conclusions drawn on this basis), and we call them "coherence properties". They are very important, not only for working with a logic which respects them, but also for soundness and completeness questions, often they are at the core of such problems.

\section{Remark 6.1}

$(R \uparrow)$ corresponds to $(I \downarrow)$ and $(F \uparrow)$ : If $A$ is small in $X \subseteq Y$, then it will a fortiori be small in the bigger $Y$.

$(R \downarrow)$ says that diminishing base sets by a small amount will keep small subsets small. This goes in the wrong direction, so we have to be careful. We cannot diminish arbitrarily, e.g., if $A$ is a small subset of $B, A$ should not be a small subset of $B-(B-A)=A$. It still seems quite safe, if "small" is a robust notion, i.e. defined in an abstract way, and not anew for each set, and, if "small" is sufficiently far from "big", as, for example in a filter.

There is, however, an important conceptual distinction to make here. Filters express "size" in an abstract way, in the context of nonmonotonic logics, $\alpha \sim \beta$ iff the set of $\alpha \wedge \neg \beta$ is small in $\alpha$. But here, we were interested in "small" changes in the reference set $X$ (or $\alpha$ in our example). So we have two quite different uses of "size", one for nonmonotonic logics, abstractly expressed by a filter, the other for coherence conditions. It is possible, but not necessary, to consider both essentially the same notions. But we should not forget that we have two conceptually different uses of size here.

$(R \downarrow \downarrow)$ is obviously a stronger variant of $(R \downarrow)$.

It and its strength is perhaps best understood as transitivity of the relation ". $\in \mathcal{M}^{+}(.)^{\prime \prime}$.

Now, (in comparison to $(R \downarrow)) A^{\prime}$ can be a medium size subset of $B$. As a matter of fact, $(R \downarrow \downarrow)$ is a very big strengthening of $(R \downarrow)$ : Consider a principal filter $\mathcal{F}:=\{X \subseteq B$ : $\left.B^{\prime} \subseteq X\right\}, b \in B^{\prime}$. Then $\{b\}$ has at least medium size, so any small set $A \subseteq B$ is smaller than $\{b\}$ - and this is, of course, just rankedness. If we only have $(R \downarrow)$, then we need the whole generating set $B^{\prime}$ to see that $A$ is small. This is the strong substitution property of rankedness: any $b$ as above will show that $A$ is small.

The more we see size as an abstract notion, and the more we see "small" different from "big" (or "medium"), the more we can go from one base set to another and find the same sizes - the more we have coherence when we reason with small and big subsets. $(R \downarrow)$ works with iterated use of "small", just as do filters, but not weak filters. So it is not surprising that weak filters and $(R \downarrow)$ do not cooperate well: Let $A, B, C$ be small subsets of $X$ - pairwise disjoint, and $A \cup B \cup C=X$, this is possible. By $(R \downarrow) B$ and $C$ will be small in $X-A$, so again by $(R \downarrow) C$ will be small in $(X-A)-B=C$, but this is absurd.

If we think that filters are too strong, but we still want some coherence, i.e. abstract size, we can consider $(R \cup d i s j)$ : If $A$ is a small subset of $B$, and $A^{\prime}$ of $B^{\prime}$, and $B$ and $B^{\prime}$ 
are disjoint, then $A \cup A^{\prime}$ is a small subset of $B \cup B^{\prime}$. It expresses a uniform approach to size, or distributivity, if you like. It holds, e.g. when we consider a set to be small iff it is smaller than a certain fraction. The important point is here that by disjointness, the big subsets do not get "used up". (This property generalizes in a straightforward way to the infinite case.)

\section{Fact 6.2}

The two versions of $(R \downarrow)$ and the three versions of $(R \downarrow \downarrow)$ are each equivalent. For the third version of $(R \downarrow \downarrow)$ we use $(I \downarrow)$.

\section{Proof}

For $A, B \subseteq X,(X-B)-((X-A)-B)=A-\mathrm{B}$.

" $\Rightarrow$ ": Let $A \in \mathcal{F}(X), B \in \mathcal{I}(X)$, so $X-A \in \mathcal{I}(X)$, so by prerequisite $(X-A)-B \in$ $\mathcal{I}(X-\mathrm{B})$, so $A-B=(X-B)-((X-A)-B) \in \mathcal{F}(X-\mathrm{B})$.

" $\Leftarrow$ ": Let $A, B \in \mathcal{I}(X)$, so $X-A \in \mathcal{F}(X)$, so by prerequisite $(X-A)-B \in \mathcal{F}(X-\mathrm{B})$, so $A-B=(X-B)-((X-A)-B) \in \mathcal{I}(X-\mathrm{B})$.

The proof for $(R \downarrow \downarrow)$ is the same for the first two cases.

It remains to show equivalence with the last one. We assume closure under set difference and union.

$(1) \Rightarrow(3)$ :

Suppose $A \notin \mathcal{M}^{+}(Y)$, but $X \in \mathcal{M}^{+}(Y)$, we show $A \notin \mathcal{M}^{+}(X)$. So $A \in \mathcal{I}(Y), Y-X \notin$ $\mathcal{F}(Y)$, so $A=A-(Y-X) \in \mathcal{I}(Y-(Y-X))=\mathcal{I}(X)$.

$(3) \Rightarrow(1)$ :

Suppose $A-B \notin \mathcal{I}(X-\mathrm{B}), B \notin \mathcal{F}(X)$, we show $A \notin \mathcal{I}(X)$. By prerequisite $A-B \in$ $\mathcal{M}^{+}(X-\mathrm{B}), X-B \in \mathcal{M}^{+}(X)$, so $A-B \in \mathcal{M}^{+}(X)$, so by $(I \downarrow) A \in \mathcal{M}^{+}(X)$, so $A \notin \mathcal{I}(X)$.

\section{Fact 6.3}

If $f(X)$ is the smallest $A$ s.t. $A \in \mathcal{F}(X)$, then, given the property on the left, the one on the right follows.

Conversely, when we define $\mathcal{F}(X):=\left\{X^{\prime}: f(X) \subseteq X^{\prime} \subseteq X\right\}$, given the property on the right, the one on the left follows. For this direction, we assume that we can use the full powerset of some base set $U$ - as is the case for the model sets of a finite language. This is 
perhaps not too bold, as we mainly want to stress here the intuitive connections, without putting too much weight on definability questions.

\begin{tabular}{|c|c|c|c|}
\hline$(1.1)$ & \multirow[t]{2}{*}{$(R \uparrow)$} & $\Rightarrow$ & \multirow[t]{2}{*}{$(\mu w O R)$} \\
\hline$(1.2)$ & & $\Leftarrow$ & \\
\hline (2.1) & \multirow[t]{2}{*}{$(R \uparrow)+(I \cup)$} & $\Rightarrow$ & \multirow[t]{2}{*}{$(\mu O R)$} \\
\hline$(2.2)$ & & $\Leftarrow$ & \\
\hline (3.1) & \multirow[t]{2}{*}{$(R \uparrow)+(I \cup)$} & $\Rightarrow$ & \multirow[t]{2}{*}{$(\mu P R)$} \\
\hline$(3.2)$ & & $\Leftarrow$ & \\
\hline$(4.1)$ & \multirow[t]{2}{*}{$(R \cup d i s j)$} & $\Rightarrow$ & \multirow[t]{2}{*}{$\overline{(\mu d i s j O R)}$} \\
\hline$(4.2)$ & & $\Leftarrow$ & \\
\hline$(5.1)$ & \multirow[t]{2}{*}{$(R \downarrow)$} & $\Rightarrow$ & \multirow[t]{2}{*}{$(\mu C M)$} \\
\hline$(5.2)$ & & $\Leftarrow$ & \\
\hline (6.1) & \multirow[t]{2}{*}{$(R \downarrow \downarrow)$} & $\Rightarrow$ & \multirow[t]{2}{*}{$(\mu$ RatM $)$} \\
\hline$(6.2)$ & & $\Leftarrow$ & \\
\hline
\end{tabular}

\section{Proof}

(1.1) $(R \uparrow) \Rightarrow(\mu w O R)$ :

$X-f(X)$ is small in $X$, so it is small in $X \cup Y$ by $(R \uparrow)$, so $A:=X \cup Y-(X-f(X)) \in$ $\mathcal{F}(X \cup Y)$, but $A \subseteq f(X) \cup Y$, and $f(X \cup Y)$ is the smallest element of $\mathcal{F}(X \cup Y)$.

$(1.2)(\mu w O R) \Rightarrow(R \uparrow):$

Let $X \subseteq Y, X^{\prime}:=Y-\mathrm{X}$. Let $A \in \mathcal{I}(X)$, so $X-A \in \mathcal{F}(X)$, so $f(X) \subseteq X-\mathrm{A}$, so $f\left(X \cup X^{\prime}\right) \subseteq f(X) \cup X^{\prime} \subseteq(X-A) \cup X^{\prime}$ by prerequisite, so $\left(X \cup X^{\prime}\right)-\left((X-A) \cup X^{\prime}\right)=$ $A \in \mathcal{I}\left(X \cup X^{\prime}\right)$.

$(2.1)(R \uparrow)+(I \cup) \Rightarrow(\mu O R):$

$X-f(X)$ is small in $X, Y-f(Y)$ is small in $Y$, so both are small in $X \cup Y$ by $(R \uparrow)$, so $A:=(X-f(X)) \cup(Y-f(Y))$ is small in $X \cup Y$ by $(I \cup)$, but $X \cup Y-(f(X) \cup f(Y)) \subseteq A$, so $f(X) \cup f(Y) \in \mathcal{F}(X \cup Y)$, so, as $f(X \cup Y)$ is the smallest element of $\mathcal{F}(X \cup Y)$, $f(X \cup Y) \subseteq f(X) \cup f(Y)$.

$(2.2)(\mu O R) \Rightarrow(R \uparrow)+(I \cup):$

Let again $X \subseteq Y, X^{\prime}:=Y-\mathrm{X}$. Let $A \in \mathcal{I}(X)$, so $X-A \in \mathcal{F}(X)$, so $f(X) \subseteq X-\mathrm{A}$. $f\left(X^{\prime}\right) \subseteq X^{\prime}$, so $f\left(X \cup X^{\prime}\right) \subseteq f(X) \cup f\left(X^{\prime}\right) \subseteq(X-A) \cup X^{\prime}$ by prerequisite, so $\left(X \cup X^{\prime}\right)$ $\left((X-A) \cup X^{\prime}\right)=A \in \mathcal{I}\left(X \cup X^{\prime}\right)$.

$(I \cup)$ holds by definition.

$(3.1)(R \uparrow)+(I \cup) \Rightarrow(\mu P R):$

Let $X \subseteq Y . Y-f(Y)$ is the largest element of $\mathcal{I}(Y), X-f(X) \in \mathcal{I}(X) \subseteq \mathcal{I}(Y)$ by $(R \uparrow)$, so $(X-f(X)) \cup(Y-f(Y)) \in \mathcal{I}(Y)$ by $(I \cup)$, so by "largest" $X-f(X) \subseteq Y-f(Y)$, so $f(Y) \cap X \subseteq f(X)$.

$(3.2)(\mu P R) \Rightarrow(R \uparrow)+(I \cup)$ 
Let again $X \subseteq Y, X^{\prime}:=Y-\mathrm{X}$. Let $A \in \mathcal{I}(X)$, so $X-A \in \mathcal{F}(X)$, so $f(X) \subseteq X-\mathrm{A}$, so by prerequisite $f(Y) \cap X \subseteq X-\mathrm{A}$, so $f(Y) \subseteq X^{\prime} \cup(X-\mathrm{A})$, so $\left(X \cup X^{\prime}\right)-\left(X^{\prime} \cup(X-A)\right)=$ $A \in \mathcal{I}(Y)$.

Again, $(I \cup)$ holds by definition.

(4.1) $(R \cup \operatorname{disj}) \Rightarrow(\mu d i s j O R)$ :

If $X \cap Y=\emptyset$, then (1) $A \in \mathcal{I}(X), B \in \mathcal{I}(Y) \Rightarrow A \cup B \in \mathcal{I}(X \cup Y)$ and $(2) A \in \mathcal{F}(X), B \in$ $\mathcal{F}(Y) \Rightarrow A \cup B \in \mathcal{F}(X \cup Y)$ are equivalent. (By $X \cap Y=\emptyset,(X-A) \cup(Y-B)=(X \cup Y)-$ $(A \cup B)$.) So $f(X) \in \mathcal{F}(X), f(Y) \in \mathcal{F}(Y) \Rightarrow$ (by prerequisite) $f(X) \cup f(Y) \in \mathcal{F}(X \cup Y)$. $f(X \cup Y)$ is the smallest element of $\mathcal{F}(X \cup Y)$, so $f(X \cup Y) \subseteq f(X) \cup f(Y)$.

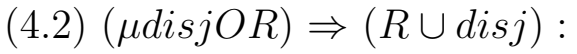

Let $X \subseteq Y, X^{\prime}:=Y-\mathrm{X}$. Let $A \in \mathcal{I}(X), A^{\prime} \in \mathcal{I}\left(X^{\prime}\right)$, so $X-A \in \mathcal{F}(X), X^{\prime}-A^{\prime} \in \mathcal{F}\left(X^{\prime}\right)$, so $f(X) \subseteq X-\mathrm{A}, f\left(X^{\prime}\right) \subseteq X^{\prime}-A^{\prime}$, so $f\left(X \cup X^{\prime}\right) \subseteq f(X) \cup f\left(X^{\prime}\right) \subseteq(X-A) \cup\left(X^{\prime}-A^{\prime}\right)$ by prerequisite, so $\left(X \cup X^{\prime}\right)-\left((X-A) \cup\left(X^{\prime}-A^{\prime}\right)\right)=A \cup A^{\prime} \in \mathcal{I}\left(X \cup X^{\prime}\right)$.

$(5.1)(R \downarrow) \Rightarrow(\mu C M)$ :

$f(X) \subseteq Y \subseteq X \Rightarrow X-Y \in \mathcal{I}(X), X-f(X) \in \mathcal{I}(X) \Rightarrow_{(R \downarrow)} A:=(X-f(X))-(X-Y) \in$ $\mathcal{I}(Y) \Rightarrow Y-A=f(X)-(X-Y) \in \mathcal{F}(Y) \Rightarrow f(Y) \subseteq f(X)-(X-Y) \subseteq f(X)$.

(5.2) $(\mu C M) \Rightarrow(R \downarrow)$

Let $A \in \mathcal{F}(X), B \in \mathcal{I}(X)$, so $f(X) \subseteq X-B \subseteq X$, so by prerequisite $f(X-B) \subseteq f(X)$. As $A \in \mathcal{F}(X), f(X) \subseteq A$, so $f(X-B) \subseteq f(X) \subseteq A \cap(X-B)=A-\mathrm{B}$, and $A-B \in \mathcal{F}(X-\mathrm{B})$.

$(6.1)(R \downarrow \downarrow) \Rightarrow(\mu R a t M):$

Let $X \subseteq Y, X \cap f(Y) \neq \emptyset$. If $Y-X \in \mathcal{F}(Y)$, then $A:=(Y-X) \cap f(Y) \in \mathcal{F}(Y)$, but by $X \cap f(Y) \neq \emptyset A \subset f(Y)$, contradicting "smallest" of $f(Y)$. So $Y-X \notin \mathcal{F}(Y)$, and by $(R \downarrow \downarrow) X-f(Y)=(Y-f(Y))-(Y-X) \in \mathcal{I}(X)$, so $X \cap f(Y) \in \mathcal{F}(X)$, so $f(X) \subseteq f(Y) \cap X$.

(6.2) $(\mu R a t M) \Rightarrow(R \downarrow \downarrow)$

Let $A \in \mathcal{F}(Y), B \notin \mathcal{F}(Y) . B \notin \mathcal{F}(Y) \Rightarrow Y-B \notin \mathcal{I}(Y) \Rightarrow(Y-B) \cap f(Y) \neq \emptyset$. Set $X:=Y-\mathrm{B}$, so $X \cap f(Y) \neq \emptyset, X \subseteq Y$, so $f(X) \subseteq f(Y) \cap X$ by prerequisite. $f(Y) \subseteq A \Rightarrow$ $f(X) \subseteq f(Y) \cap X=f(Y)-B \subseteq A-\mathrm{B}$.

\section{Definition 6.2}

Augment the language of first order logic by the new quantifier: If $\phi$ and $\psi$ are formulas, then so are $\nabla x \phi(x), \nabla x \phi(x): \psi(x)$, for any variable $x$. The:-versions are the restricted variants. We call any formula of $\mathcal{L}$, possibly containing $\nabla$ a $\nabla-\mathcal{L}$-formula. 


\section{Definition 6.3}

$(\mathcal{N}-$ Model $)$

Let $\mathcal{L}$ be a first order language, and $M$ be a $\mathcal{L}$-structure. Let $\mathcal{N}(M)$ be a weak filter, or $\mathcal{N}$-system - $\mathcal{N}$ for normal - over $M$. Define $\langle M, \mathcal{N}(M)>\models \phi$ for any $\nabla-\mathcal{L}$-formula inductively as usual, with one additional induction step:

$<M, \mathcal{N}(M)>\models \nabla x \phi(x)$ iff there is $A \in \mathcal{N}(M)$ s.t. $\forall a \in A(<M, \mathcal{N}(M)>\models \phi[a])$.

\section{Definition 6.4}

Let any axiomatization of predicate calculus be given. Augment this with the axiom schemata

(1) $\nabla x \phi(x) \wedge \forall x(\phi(x) \rightarrow \psi(x)) \rightarrow \nabla x \psi(x)$,

(2) $\nabla x \phi(x) \rightarrow \neg \nabla x \neg \phi(x)$,

(3) $\forall x \phi(x) \rightarrow \nabla x \phi(x)$ and $\nabla x \phi(x) \rightarrow \exists x \phi(x)$,

(4) $\nabla x \phi(x) \leftrightarrow \nabla y \phi(y)$ if $x$ does not occur free in $\phi(y)$ and $y$ does not occur free in $\phi(x)$. (for all $\phi, \psi)$.

\section{Proposition 6.4}

The axioms given in Definition 6.4 are sound and complete for the semantics of Definition 6.3

\section{Definition 6.5}

Call $\mathcal{N}^{+}(M)=<\mathcal{N}(N): N \subseteq M>$ a $\mathcal{N}^{+}-$system or system of weak filters over $M$ iff for each $N \subseteq M \mathcal{N}(N)$ is a weak filter or $\mathcal{N}$-system over $N$. (It suffices to consider the definable subsets of $M$.)

\section{Definition 6.6}

Let $\mathcal{L}$ be a first order language, and $M$ a $\mathcal{L}$-structure. Let $\mathcal{N}^{+}(M)$ be a $\mathcal{N}^{+}-$system over $M$.

Define $<M, \mathcal{N}^{+}(M)>\models \phi$ for any formula inductively as usual, with the additional induction steps:

1. $\left\langle M, \mathcal{N}^{+}(M)>\models \nabla x \phi(x)\right.$ iff there is $A \in \mathcal{N}(M)$ s.t. $\forall a \in A\left(<M, \mathcal{N}^{+}(M)>\models\right.$ $\phi[a])$,

2. $<M, \mathcal{N}^{+}(M)>\models \nabla x \phi(x): \psi(x)$ iff there is $A \in \mathcal{N}\left(\left\{x:<M, \mathcal{N}^{+}(M)>\models \phi(x)\right\}\right)$ s.t. $\forall a \in A\left(<M, \mathcal{N}^{+}(M)>\models \psi[a]\right)$. 


\section{Definition 6.7}

Extend the logic of first order predicate calculus by adding the axiom schemata

(1) a. $\nabla x \phi(x) \leftrightarrow \nabla x(x=x): \phi(x), b . \forall x(\sigma(x) \leftrightarrow \tau(x)) \wedge \nabla x \sigma(x): \phi(x) \rightarrow \nabla x \tau(x)$ : $\phi(x)$,

(2) $\nabla x \phi(x): \psi(x) \wedge \forall x(\phi(x) \wedge \psi(x) \rightarrow \vartheta(x)) \rightarrow \nabla x \phi(x): \vartheta(x)$,

(3) $\exists x \phi(x) \wedge \nabla x \phi(x): \psi(x) \rightarrow \neg \nabla x \phi(x): \neg \psi(x)$,

(4) $\forall x(\phi(x) \rightarrow \psi(x)) \rightarrow \nabla x \phi(x): \psi(x)$ and $\nabla x \phi(x): \psi(x) \rightarrow[\exists x \phi(x) \rightarrow \exists x(\phi(x) \wedge \psi(x))]$,

(5) $\nabla x \phi(x): \psi(x) \leftrightarrow \nabla y \phi(y): \psi(y)$ (under the usual caveat for substitution).

(for all $\phi, \psi, \vartheta, \sigma, \tau)$.

\section{Proposition 6.5}

The axioms of Definition 6.7 are sound and complete for the $\mathcal{N}^{+}-$semantics of $\nabla$ as defined in Definition 6.6.

\section{References}

[AGM85] C.Alchourron, P.Gardenfors, D.Makinson, "On the Logic of Theory Change: partial meet contraction and revision functions", Journal of Symbolic Logic, Vol. 50, pp. 510-530, 1985

[KLM90] S.Kraus, D.Lehmann, M.Magidor, "Nonmonotonic reasoning, preferential models and cumulative logics", Artificial Intelligence, 44 (1-2), p.167-207, July 1990

[Sch92] K.Schlechta: "Some results on classical preferential models", Journal of Logic and Computation, Oxford, Vol.2, No.6 (1992), p. 675-686 